\title{
ON THE SOURCE OF THE DUST EXTINCTION IN TYPE Ia SUPERNOVAE AND THE DISCOVERY OF ANOMALOUSLY STRONG Na I ABSORPTION*
}

\author{
M. M. Phillips ${ }^{1}$, Joshua D. Simon ${ }^{2}$, Nidia Morrell ${ }^{1}$, Christopher R. Burns ${ }^{2}$, Nick L. J. Cox ${ }^{3}$, Ryan J. Foley ${ }^{4}$,

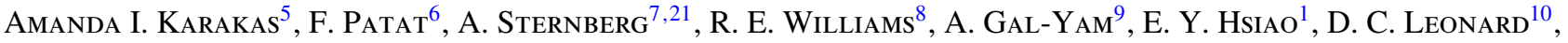 \\ Sven E. Persson $^{2}$, Maximilian Stritzinger ${ }^{11}$, I. B. Thompson ${ }^{2}$, Abdo Campillay ${ }^{1}$, Carlos Contreras $^{1}$, \\ Gastón Folatelli $^{12}$, Wendy L. Freedman ${ }^{2}$, Mario Hamuy ${ }^{13}$, Miguel Roth ${ }^{1}$, Gregory A. Shields ${ }^{14}$, \\ Nicholas B. SuntzefF ${ }^{15}$, Laura Chomiuk ${ }^{4}$, Inese I. Ivans ${ }^{16}$, Barry F. Madore ${ }^{2,17}$, B. E. Penprase ${ }^{18}$, \\ Daniel Perley ${ }^{19}$, G. Pignata ${ }^{20}$, G. Preston ${ }^{2}$, and Alicia M. Soderberg $^{4}$ \\ ${ }^{1}$ Carnegie Observatories, Las Campanas Observatory, Casilla 601, La Serena, Chile; mmp@ @ lco.cl \\ ${ }^{2}$ Observatories of the Carnegie Institution for Science, 813 Santa Barbara St., Pasadena, CA 91101, USA \\ ${ }^{3}$ Instituut voor Sterrenkunde, KU Leuven, Celestijnenlaan 200D bus 2401, 3001 Leuven, Belgium \\ ${ }^{4}$ Harvard-Smithsonian Center for Astrophysics, 60 Garden Street, Cambridge, MA 02138, USA \\ ${ }^{5}$ Research School of Astronomy and Astrophysics, The Australian National University, Weston, ACT 2611, Australia \\ ${ }^{6}$ European Southern Observatory (ESO), Karl Schwarschild Strasse 2, D-85748, Garching bei München, Germany \\ ${ }^{7}$ Max Planck Institute for Astrophysics, Karl Schwarzschild Strasse 1, D-85741 Garching bei München, Germany \\ ${ }^{8}$ Space Telescope Science Institute, 3700 San Martin Drive, Baltimore, MD 21218, USA \\ ${ }^{9}$ Benoziyo Center for Astrophysics, Faculty of Physics, Weizmann Institute of Science, Rehovot 76100, Israel \\ ${ }^{10}$ Department of Astronomy, San Diego State University, San Diego, CA 92182, USA \\ ${ }^{11}$ Department of Physics and Astronomy, Aarhus University, Ny Munkegade 120, DK-8000 Aarhus C, Denmark \\ ${ }^{12}$ Kavli Institute for the Physics and Mathematics of the Universe, Todai Institutes for Advanced Study, \\ the University of Tokyo, Kashiwa 277-8583, Japan \\ ${ }^{13}$ Universidad de Chile, Departamento de Astronomía, Casilla 36-D, Santiago, Chile \\ ${ }^{14}$ Department of Astronomy, University of Texas, Austin, TX 78712, USA \\ ${ }^{15}$ George P. and Cynthia Woods Mitchell Institute for Fundamental Physics and Astronomy, Texas A\&M University, \\ Department of Physics and Astronomy, College Station, TX 77843, USA \\ ${ }^{16}$ Department of Physics and Astronomy, University of Utah, Salt Lake City, UT 84112, USA \\ ${ }^{17}$ Infrared Processing and Analysis Center, Caltech/Jet Propulsion Laboratory, Pasadena, CA 91125, USA \\ ${ }^{18}$ Department of Physics and Astronomy, Pomona College, 610 N. College Ave., Claremont, CA 91711, USA \\ ${ }^{19}$ Cahill Center for Astrophysics, California Institute of Technology, Pasadena, CA 91125, USA \\ ${ }^{20}$ Departamento de Ciencias Fisicas, Universidad Andres Bello, Avda. Republica 252, Santiago, Chile \\ Received 2013 July 11; accepted 2013 November 1; published 2013 November 22
}

\begin{abstract}
High-dispersion observations of the $\mathrm{Na}$ I $\mathrm{D} \lambda \lambda 5890,5896$ and $\mathrm{K}_{\mathrm{I}} \lambda \lambda 7665,7699$ interstellar lines, and the diffuse interstellar band at $5780 \AA$ in the spectra of 32 Type Ia supernovae are used as an independent means of probing dust extinction. We show that the dust extinction of the objects where the diffuse interstellar band at $5780 \AA$ is detected is consistent with the visual extinction derived from the supernova colors. This strongly suggests that the dust producing the extinction is predominantly located in the interstellar medium of the host galaxies and not in circumstellar material associated with the progenitor system. One quarter of the supernovae display anomalously large Na I column densities in comparison to the amount of dust extinction derived from their colors. Remarkably, all of the cases of unusually strong Na I D absorption correspond to "Blueshifted" profiles in the classification scheme of Sternberg et al. This coincidence suggests that outflowing circumstellar gas is responsible for at least some of the cases of anomalously large $\mathrm{Na}$ I column densities. Two supernovae with unusually strong Na I D absorption showed essentially normal $\mathrm{K}$ I column densities for the dust extinction implied by their colors, but this does not appear to be a universal characteristic. Overall, we find the most accurate predictor of individual supernova extinction to be the equivalent width of the diffuse interstellar band at $5780 \AA$, and provide an empirical relation for its use. Finally, we identify ways of producing significant enhancements of the $\mathrm{Na}$ abundance of circumstellar material in both the single-degenerate and double-degenerate scenarios for the progenitor system.
\end{abstract}

Key words: circumstellar matter - dust, extinction - galaxies: ISM - supernovae: general

Online-only material: color figures

\section{INTRODUCTION}

Type Ia supernovae (SNe Ia) are one of the most effective observational tools for measuring the expansion history of the universe. Their successful use in cosmology is due to the discovery of empirical relations that dramatically decrease the dispersion in peak luminosities at optical wavelengths. The first

\footnotetext{
* This paper includes data gathered with the $6.5 \mathrm{~m}$ Magellan telescopes at Las Campanas Observatory, Chile.

${ }^{21}$ Minerva Fellow.
}

of these is the well-known correlation with light curve shape: intrinsically brighter $\mathrm{SNe}$ Ia have broader light curves that decline more slowly from maximum than do the light curves of less luminous SNe Ia (Phillips 1993). The second is a strong dependence of peak luminosity on color that is in the same sense as dust reddening, but with an average value of the ratio of total-to-selective extinction, $R_{V}$, that is significantly less than would be produced by normal interstellar dust in the Milky Way (Tripp 1998). The latter result has variously been interpreted as possible evidence that the extinction arises in circumstellar 
dust (Wang 2005; Goobar 2008), as the consequence of intrinsic differences in color between SNe Ia with "normal" and "high" Si II expansion velocities (Foley \& Kasen 2011), or as a bias due to a misidentification of the dispersion in the luminosity/colorcorrected Hubble diagram with an intrinsic scatter in luminosity rather than color (Scolnic et al. 2013).

Our understanding of the progenitors and explosion mechanism(s) that produce SNe Ia is still quite limited. Although there is widespread agreement that these objects correspond to the thermonuclear disruption of a white dwarf in a binary system, it is not yet clear if the companion to the white dwarf is a main sequence or giant star ("single-degenerate" or "SD" model) or another white dwarf ("double-degenerate" or "DD" model). In recent years, observational evidence favoring both scenarios has been put forward (e.g., see Howell 2011; Maoz \& Mannucci 2012; Patat 2013). In both the SD and DD scenarios, material ejected from the system prior to the explosion may remain as circumstellar material (CSM; Moore \& Bildsten 2012; Raskin \& Kasen 2013; Shen et al. 2013). Sternberg et al. (2011) found a strong statistical preference for blueshifted structures in the narrow $\mathrm{Na}$ I D absorption observed in the line-of-sight to many SNe Ia, suggestive of gas outflows from the progenitor systems. In a few such cases, temporal variations of blueshifted components of the Na I D lines apparently due to changing ionization conditions in the CSM have also been observed (e.g., Patat et al. 2007; Simon et al. 2009; Dilday et al. 2012). On the other hand, radio and X-ray observations of the prototypical Type Ia SN 2011fe place tight upper limits on the amount of CSM in the progenitor system before explosion (e.g., Horesh et al. 2012), and early-time photometry of this event apparently rules out either a red giant or main sequence companion (Bloom et al. 2012).

In the Milky Way, the strengths of certain interstellar absorption features such as the $\mathrm{Na}$ I D lines and the diffuse interstellar bands (DIBs) have been known for many years to correlate with dust extinction (e.g., Merrill \& Wilson 1938; Hobbs 1974). In this paper, we employ high-dispersion spectroscopy to use these features as an independent probe of the dust affecting the colors of SNe Ia. As we will show, the data indicate that the dust extinction for the objects where DIBs are observed is generally consistent with the extinction derived from the SN colors, and therefore most likely arises in the interstellar medium of the host galaxy. However, one-fourth of the SNe Ia, all with blueshifted structures as per Sternberg et al. (2011), display anomalously large Na I column densities that, in the interstellar medium (ISM) of the Milky Way, would correspond to an order of magnitude or more greater dust extinction than that implied by the SN colors.

\section{OBSERVATIONS AND ANALYSIS}

\subsection{Column Densities and Equivalent Widths}

Our approach is to first examine the relationship between dust extinction and interstellar absorption lines in the Milky Way. These results will then be contrasted with a similar comparison between the dust extinction in the line-of-sight to the SN Ia as derived from their optical and near-infrared (NIR) light curves, and the narrow absorption lines produced by the host galaxy ISM and/or a pre-existing CSM (hereafter referred to collectively as "host absorption").

In the first case, we employ a sample of $46 \mathrm{SNe}$ and active galactic nuclei (AGNs) as external beacons to study the absorption lines produced by the ISM of the Milky Way. Echelle spectra of 22 of these objects are drawn from the observations of thermonuclear and core-collapse SNe published by Sternberg et al. (2011) and available through WISeREP (Yaron \& Gal-Yam $2012^{22}$ ). The remaining 24 spectra in our data set correspond to unpublished observations of SNe and AGNs obtained with the Magellan Inamori Kyocera Echelle (MIKE; Bernstein et al. 2003) on the $6.5 \mathrm{~m}$ Clay telescope. Table 1 lists the objects in this sample. Henceforth, we refer to these objects as the "Milky Way" sample.

To study the relationship between the SN dust extinction and the narrow host absorption lines, we have put together a sample of $32 \mathrm{SNe}$ Ia with both high-dispersion spectra and well-observed light curves. Spectra for 21 of these SNe Ia were drawn from the Sternberg et al. (2011) study (also available through WISeREP), and an additional 6 are taken from Foley et al. (2012b). Results for the remaining $5 \mathrm{SNe}$ are taken from the literature. Table 2 lists the full sample of $32 \mathrm{SNe}$ Ia along with host galaxy names, morphologies, and references to the SN photometry. Table 3 gives the sources and wavelength resolutions of the high-dispersion spectral observations. These $\mathrm{SNe}$ are referred to as the "host absorption" sample in the remainder of this paper.

Column densities of neutral sodium and potassium were measured for both the Milky Way and host absorption components of the NaI D $\lambda \lambda 5890,5896$ and $\mathrm{K}_{\mathrm{I}} \lambda \lambda 7665,7699$ doublets using the Voigt profile fitting program, VPFIT, ${ }^{23}$ developed by R. F. Carswell, J. K. Webb, and others, in combination with the VPGUESS ${ }^{24}$ interface of J. Liske. Upper limits for nondetections of both $\mathrm{Na}$ I and $\mathrm{K}$ I were calculated by first estimating an upper limit to the equivalent width, and then converting this to a column density using empirical relations between equivalent width and column density derived from weak, unsaturated lines in other objects. In cases where the $\mathrm{Na}$ I $\mathrm{D}$ lines were significantly saturated $\left(\log N_{\mathrm{Na}} \gtrsim 13 \mathrm{~cm}^{-2}\right)$, the much weaker $\mathrm{K}_{\mathrm{I}}$ lines were used to determine the velocity and Doppler parameter, $b$, of each visible component, and this information was employed in fitting the saturated portion of the $\mathrm{Na}$ I $\mathrm{D}$ profiles. This procedure was possible for most of the objects observed from 2006 onward. Four of the SNe in the host absorption sample had extremely strong D lines $\left(\log N_{\mathrm{Na}}>13.5 \mathrm{~cm}^{-2}\right)$. For one of these-SN 2002bo-the spectral coverage did not include the $\mathrm{K}$ I lines. A model with two absorption components provided a significantly better fit to the severely saturated profiles of the D lines in this SN than did one with a single component, and so we have adopted the results of the two-component model in this paper. However, without the additional information provided by the $\mathrm{K}_{\mathrm{I}}$ lines, the error associated with the measurement of $\log N_{\mathrm{Na}}$ is large.

Kemp et al. (2002) found that Na I column densities measured from fitting profiles to the $\mathrm{D}$ lines for values $\log N_{\mathrm{Na}}>$ $12.5 \mathrm{~cm}^{-2}$ were systematically underestimated by $0.40-0.70 \mathrm{dex}$ compared to column densities measured from the much weaker Na I UV $\lambda \lambda 3302,3303$ doublet. The UV lines are not covered by our echelle spectra so we cannot confirm this, although as mentioned in Section 4.5, a comparison of the $N_{\mathrm{Na} \text { I }} / N_{\mathrm{K} \text { I }}$ ratio derived for our Milky Way sample with the measurements of Kemp et al. (2002) suggests that our $N_{\mathrm{Na}}$ values may be similarly affected. This should be borne in mind when using the relations involving the $\mathrm{Na}$ I column density developed in

\footnotetext{
22 http://www.weizmann.ac.il/astrophysics/wiserep/

23 http://www.ast.cam.ac.uk/ rfc/vpfit.html

24 http://www.eso.org/ jliske/vpguess/
} 
Table 1

Milky Way Na I and K I Column Density Measurements

\begin{tabular}{|c|c|c|c|c|}
\hline $\begin{array}{l}\text { Object } \\
\text { (1) }\end{array}$ & $\begin{array}{c}A_{V} \\
(\mathrm{mag}) \\
(2)\end{array}$ & $\begin{array}{c}\log N_{\mathrm{Na}} \\
\left(\mathrm{cm}^{-2}\right) \\
(3)\end{array}$ & $\begin{array}{c}\log N_{\mathrm{K}_{\mathrm{I}}} \\
\left(\mathrm{cm}^{-2}\right) \\
(4)\end{array}$ & $\begin{array}{c}\text { Reference } \\
\text { (5) }\end{array}$ \\
\hline $2003 \mathrm{gd}$ & $0.19 \pm 0.03$ & $12.775 \pm 0.034$ & $\cdots$ & 1 \\
\hline 2006be & $0.08 \pm 0.01$ & $12.068 \pm 0.038$ & $\cdots$ & 2 \\
\hline $2006 \mathrm{ca}$ & $0.64 \pm 0.10$ & $13.181 \pm 0.052$ & $\cdots$ & 2 \\
\hline $2006 \mathrm{eu}$ & $0.52 \pm 0.08$ & $12.914 \pm 0.039$ & $\cdots$ & 2 \\
\hline 2007af & $0.11 \pm 0.02$ & $11.751 \pm 0.106$ & $\cdots$ & 2 \\
\hline 2007hj & $0.26 \pm 0.04$ & $12.859 \pm 0.102$ & $\cdots$ & 2 \\
\hline $2007 \mathrm{kk}$ & $0.64 \pm 0.10$ & $12.801 \pm 0.122$ & $\cdots$ & 2 \\
\hline 2007le & $0.09 \pm 0.02$ & $11.924 \pm 0.055$ & $\cdots$ & 2 \\
\hline 2007on & $0.03 \pm 0.01$ & $11.178 \pm 0.077$ & $\cdots$ & 2 \\
\hline $2007 \mathrm{sr}$ & $0.13 \pm 0.02$ & $11.734 \pm 0.018$ & $\cdots$ & 2 \\
\hline $2008 \mathrm{C}$ & $0.23 \pm 0.04$ & $12.777 \pm 0.467$ & $\cdots$ & 2 \\
\hline $2008 f p$ & $0.54 \pm 0.09$ & $13.141 \pm 0.061$ & $11.417 \pm 0.070$ & 2 \\
\hline 2008ge & $0.04 \pm 0.01$ & $11.307 \pm 0.045$ & $\ldots$ & 2 \\
\hline $2008 \mathrm{hv}$ & $0.09 \pm 0.01$ & $12.276 \pm 0.016$ & $\cdots$ & 2 \\
\hline 2008ia & $0.62 \pm 0.10$ & $13.149 \pm 0.010$ & $11.545 \pm 0.070$ & 2 \\
\hline $2009 \mathrm{ds}$ & $0.11 \pm 0.02$ & $12.489 \pm 0.020$ & $\cdots$ & 2 \\
\hline $2009 \mathrm{ev}$ & $0.28 \pm 0.05$ & $12.737 \pm 0.040$ & $\cdots$ & 2 \\
\hline 2009iw & $0.24 \pm 0.04$ & $12.543 \pm 0.021$ & $\cdots$ & 2 \\
\hline 20091e & $0.05 \pm 0.01$ & $11.793 \pm 0.011$ & $\cdots$ & 2 \\
\hline $2009 \mathrm{mz}$ & $0.08 \pm 0.01$ & $11.972 \pm 0.030$ & $\cdots$ & 2 \\
\hline $2009 \mathrm{nr}$ & $0.07 \pm 0.01$ & $11.926 \pm 0.031$ & $\cdots$ & 2 \\
\hline 2010A & $0.08 \pm 0.01$ & $11.431 \pm 0.032$ & $\cdots$ & 2 \\
\hline $2010 \mathrm{ev}$ & $0.29 \pm 0.05$ & $12.564 \pm 0.028$ & $\cdots$ & 2 \\
\hline 2010j1 & $0.07 \pm 0.01$ & $11.791 \pm 0.119$ & $\cdots$ & 1 \\
\hline $2010 \mathrm{ko}$ & $0.39 \pm 0.06$ & $12.767 \pm 0.103$ & $11.210 \pm 0.087$ & 1 \\
\hline $2011 \mathrm{~K}$ & $0.27 \pm 0.04$ & $12.500 \pm 0.016$ & $\cdots$ & 1 \\
\hline $2011 \mathrm{di}$ & $0.29 \pm 0.05$ & $12.472 \pm 0.025$ & $\cdots$ & 1 \\
\hline $2011 d n$ & $0.49 \pm 0.08$ & $12.948 \pm 0.011$ & $11.614 \pm 0.052$ & 1 \\
\hline $2011 d q$ & $0.31 \pm 0.05$ & $12.808 \pm 0.022$ & $11.619 \pm 0.024$ & 1 \\
\hline $2011 d y$ & $0.19 \pm 0.03$ & $12.960 \pm 0.056$ & $11.103 \pm 0.041$ & 1 \\
\hline $2011 \mathrm{ek}$ & $0.97 \pm 0.15$ & $12.999 \pm 0.024$ & $11.867 \pm 0.038$ & 1 \\
\hline $2011 \mathrm{fj}$ & $0.47 \pm 0.07$ & $13.043 \pm 0.047$ & $\cdots$ & 1 \\
\hline $2012 \mathrm{cg}$ & $0.07 \pm 0.01$ & $11.218 \pm 0.057$ & $\cdots$ & 1 \\
\hline PTF11iqb & $0.09 \pm 0.02$ & $12.004 \pm 0.007$ & $\cdots$ & 1 \\
\hline $3 \mathrm{C} 273$ & $0.06 \pm 0.01$ & $12.073 \pm 0.014$ & $\cdots$ & 1 \\
\hline IC4329A & $0.16 \pm 0.02$ & $11.999 \pm 0.137$ & $\cdots$ & 1 \\
\hline Mk509 & $0.16 \pm 0.02$ & $12.142 \pm 0.041$ & $\cdots$ & 1 \\
\hline NGC 1068 & $0.09 \pm 0.02$ & $12.618 \pm 0.156$ & $\cdots$ & 1 \\
\hline NGC 2110 & $1.03 \pm 0.16$ & $13.149 \pm 0.071$ & $11.559 \pm 0.049$ & 1 \\
\hline NGC 3783 & $0.33 \pm 0.05$ & $12.656 \pm 0.013$ & $11.169 \pm 0.055$ & 1 \\
\hline PDS456 & $1.42 \pm 0.23$ & $13.309 \pm 0.037$ & $11.757 \pm 0.022$ & 1 \\
\hline Fairall51 & $0.30 \pm 0.05$ & $12.388 \pm 0.036$ & $\cdots$ & 1 \\
\hline IRAS06213+0020 & $1.77 \pm 0.28$ & $13.538 \pm 0.049$ & $12.167 \pm 0.018$ & 1 \\
\hline IRAS08311-2459 & $0.29 \pm 0.05$ & $12.668 \pm 0.040$ & $\cdots$ & 1 \\
\hline IRAS09149-6206 & $0.50 \pm 0.08$ & $12.643 \pm 0.100$ & $11.023 \pm 0.075$ & 1 \\
\hline IRAS11353-4854 & $0.53 \pm 0.08$ & $12.771 \pm 0.075$ & $11.522 \pm 0.027$ & 1 \\
\hline
\end{tabular}

Notes. Columns: (1) Object name; (2) Milky Way dust extinction (Schlafly \& Finkbeiner 2011); (3) Logarithm of the total neutral sodium column density; (4) Logarithm of the total neutral potassium column density; (5) High-dispersion spectroscopy reference [1 = unpublished MIKE spectrum; $2=$ Sternberg et al. (2011)].

Section 3.1. Nevertheless, since our approach is to compare the SN host absorption measurements relative to our same measurements for the Milky Way, this problem should not affect our conclusions.

Although the $\mathrm{Ca}$ II $\mathrm{H} \& \mathrm{~K}$ lines were also present in many of the SNe spectra, except for a few specific objects, they are not included in this study since the column density of $\mathrm{Ca}$ II is poorly correlated with dust extinction in the Milky Way, presumably due to variations in the large depletion factor of calcium (Hobbs 1974).
Equivalent widths of the DIB at $5780 \AA$ were calculated using the IRAF ${ }^{25}$ task fitprofs assuming a Gaussian profile of $2.1 \AA$ FWHM, a typical value in the Milky Way (Tuairisg et al. 2000; Welty et al. 2006; Hobbs et al. 2008). ${ }^{26}$ This feature was

\footnotetext{
25 IRAF is distributed by the National Optical Astronomy Observatory, which is operated by the Association of Universities for Research in Astronomy (AURA) under cooperative agreement with the National Science Foundation. 26 Although very high dispersion spectroscopy has shown that the profile of the 5780 A feature is not Gaussian (Galazutdinov et al. 2008), the wavelength resolution and signal-to-noise ratio of our observations do not warrant a more sophisticated method of determining the equivalent width.
} 
Table 2

SNe Ia Host Galaxy Information and Light-curve Measurements

\begin{tabular}{|c|c|c|c|c|c|c|}
\hline \multirow[b]{2}{*}{$\begin{array}{l}\text { SN } \\
\text { (1) }\end{array}$} & \multirow[b]{2}{*}{$\begin{array}{c}\text { Host Galaxy } \\
\text { (2) }\end{array}$} & \multirow[b]{2}{*}{$\begin{array}{c}\text { Morphology } \\
\text { (3) }\end{array}$} & \multicolumn{2}{|c|}{ Photometry References } & \multirow[b]{2}{*}{$\begin{array}{l}R_{V} \\
(6)\end{array}$} & \multirow{2}{*}{$\begin{array}{c}A_{V} \\
(\mathrm{mag}) \\
(7)\end{array}$} \\
\hline & & & $\begin{array}{l}\text { Optical } \\
\text { (4) }\end{array}$ & $\begin{array}{c}\text { Near-IR } \\
\text { (5) }\end{array}$ & & \\
\hline 1986G & NGC 5128 & S0 pec & 1 & 16 & $2.57_{-0.21}^{+0.23}$ & $2.03_{-0.13}^{+0.09}$ \\
\hline 1994D & NGC 4526 & $\mathrm{SAB}(\mathrm{s}) 0^{\wedge} 0 ?(\mathrm{~s})$ & 2 & 17 & $2.24_{-0.73}^{+0.62}$ & $<0.08$ \\
\hline 2001cp & UGC 10738 & $\mathrm{Sbc}$ & 3 & $\ldots$ & $2.28_{-0.80}^{+0.62}$ & $<0.17$ \\
\hline $2001 \mathrm{da}$ & NGC 7780 & $\mathrm{Sab}$ & 3 & $\cdots$ & $1.82_{-0.53}^{+0.76}$ & $0.49_{-0.14}^{+0.19}$ \\
\hline 2001el & NGC 1448 & SAcd? edge-on & 4 & 4 & $2.25_{-0.36}^{+0.46}$ & $0.62_{-0.08}^{+0.08}$ \\
\hline 2002bo & NGC 3190 & SA(s)a pec edge-on & 5 & 5 & $1.22_{-0.21}^{+0.26}$ & $0.62_{-0.10}^{+0.09}$ \\
\hline $2002 \mathrm{cr}$ & NGC 5468 & $\mathrm{SAB}(\mathrm{rs}) \mathrm{cd}$ & 6 & .. & $2.16_{-0.68}^{+0.66}$ & $0.21_{-0.07}^{+0.09}$ \\
\hline 2002ha & NGC 6962 & $\mathrm{SAB}(\mathrm{r}) \mathrm{ab}$ & 6 & $\cdots$ & $2.45_{-0.77}^{+0.52}$ & $<0.08$ \\
\hline $2002 \mathrm{jg}$ & NGC 7253B & S? & 3 & $\cdots$ & $2.46_{-0.63}^{+0.57}$ & $<0.17$ \\
\hline $2006 \mathrm{X}$ & NGC 4321 & $\mathrm{SAB}(\mathrm{s}) \mathrm{bc}$ & 7 & 7 & $1.31_{-0.10}^{+0.08}$ & $1.88_{-0.13}^{+0.09}$ \\
\hline $2006 \mathrm{~cm}$ & UGC 11723 & Sb edge-on & 4 & $\cdots$ & $1.95_{-0.33}^{+0.46}$ & $2.15_{-0.35}^{+0.40}$ \\
\hline 2007af & NGC 5584 & $\mathrm{SAB}(\mathrm{rs}) \mathrm{cd}$ & 8 & 8 & $2.11_{-0.48}^{+0.55}$ & $0.31_{-0.06}^{+0.06}$ \\
\hline $2007 \mathrm{fb}$ & UGC 12859 & $\mathrm{Sbc}$ & 9 & $\cdots$ & $2.17_{-0.60}^{+0.53}$ & $<0.09$ \\
\hline $2007 \mathrm{fs}$ & ESO 601-G005 & $\mathrm{Sb} ?$ & 9 & $\cdots$ & $2.24_{-0.78}^{+0.64}$ & $0.12_{-0.05}^{+0.08}$ \\
\hline 2007hj & NGC 7461 & SB0 & 10 & 10 & $1.96_{-0.55}^{+0.59}$ & $<0.13$ \\
\hline $2007 \mathrm{kk}$ & UGC 2828 & $\mathrm{SB}(\mathrm{rs}) \mathrm{bc}$ & 9 & $\ldots$ & $2.13_{-0.64}^{+0.55}$ & $<0.04$ \\
\hline 2007le & NGC 7721 & $\mathrm{SA}(\mathrm{s}) \mathrm{c}$ & 8 & 8 & $1.46_{-0.24}^{+0.32}$ & $0.54_{-0.08}^{+0.08}$ \\
\hline 2007on & NGC 1404 & E1 & 8 & 8 & $1.93_{-0.61}^{+0.80}$ & $<0.03$ \\
\hline $2007 \mathrm{sr}$ & NGC 4038 & $\mathrm{SB}(\mathrm{s}) \mathrm{m}$ pec & 11 & 11 & $1.74_{-0.64}^{+0.50}$ & $0.23_{-0.07}^{+0.06}$ \\
\hline $2008 \mathrm{C}$ & UGC 3611 & $\mathrm{~S} 0 / \mathrm{a}$ & 8 & 8 & $2.42_{-0.72}^{+0.56}$ & $0.39_{-0.11}^{+0.16}$ \\
\hline $2008 \mathrm{ec}$ & NGC 7469 & $\left(\mathrm{R}^{\prime}\right) \mathrm{SAB}(\mathrm{rs}) \mathrm{a}$ & 10 & $\cdots$ & $2.21_{-0.73}^{+0.53}$ & $0.53_{-0.16}^{+0.13}$ \\
\hline $2008 f \mathrm{fp}$ & ESO 428-G014 & SAB0^0(r) pec & 8 & 8 & $1.20_{-0.14}^{+0.26}$ & $0.71_{-0.08}^{+0.10}$ \\
\hline $2008 \mathrm{hv}$ & NGC 2765 & S0 & 8 & 8 & $2.23_{-0.68}^{+0.63}$ & $<0.08$ \\
\hline $2008 \mathrm{ia}$ & ESO 125-G006 & S0 & 8 & 8 & $2.35_{-0.57}^{+0.62}$ & $0.11_{-0.03}^{+0.05}$ \\
\hline SNF20080514-002 & UGC 8472 & S0 & 3 & $\cdots$ & $2.03_{-0.58}^{+0.66}$ & $<0.02$ \\
\hline $2009 \mathrm{ds}$ & NGC 3905 & $\mathrm{SB}(\mathrm{rs}) \mathrm{c}$ & 10 & 10 & $2.20_{-0.69}^{+0.55}$ & $0.16_{-0.05}^{+0.08}$ \\
\hline 2009ig & NGC 1015 & $\mathrm{SB}(\mathrm{r}) \mathrm{a}$ ? & 12 & $\cdots$ & $2.29_{-0.62}^{+0.68}$ & $<0.05$ \\
\hline 2009le & ESO 478-G006 & $\mathrm{Sbc}$ & 10 & 10 & $2.48_{-0.83}^{+0.57}$ & $0.24_{-0.08}^{+0.09}$ \\
\hline $2010 \mathrm{~A}$ & UGC 2019 & S? & 9 & $\cdots$ & $2.24_{-0.74}^{+0.75}$ & $0.39_{-0.11}^{+0.14}$ \\
\hline $2010 \mathrm{ev}$ & NGC 3244 & $\mathrm{SA}(\mathrm{rs}) \mathrm{cd}$ & 13 & $\cdots$ & $1.54_{-0.59}^{+0.57}$ & $0.50_{-0.19}^{+0.17}$ \\
\hline $2011 \mathrm{fe}$ & NGC 5457 & $\mathrm{SAB}(\mathrm{rs}) \mathrm{cd}$ & 14 & 18 & $1.63_{-0.53}^{+0.60}$ & $0.15_{-0.06}^{+0.19}$ \\
\hline $2012 \mathrm{cg}$ & NGC 4424 & $\mathrm{SB}(\mathrm{s}) \mathrm{a} ?$ & 15 & 15 & $2.7 \pm 0.5$ & $0.67 \pm 0.12$ \\
\hline
\end{tabular}

Notes. Columns: (1) SN name; (2) Host galaxy name; (3) Host galaxy morphology from the NASA/IPAC Extragalactic Database (NED); (4) Optical photometry reference; (5) Near-infrared photometry reference; (6) Host dust extinction $R_{V}$ value; (7) Host dust extinction $A_{V}$ value.

References. (1) Phillips et al. 1987; (2) CTIO 0.9 m unpublished; (3) Ganeshalingam et al. 2010; (4) Hicken et al. 2009; (5) Krisciunas et al. 2004; (6) Krisciunas et al. 2003; (7) Contreras et al. 2010; (8) Stritzinger et al. 2011; (9) Hicken et al. 2012; (10) CSP, unpublished; (11) Schweizer et al. 2008; (12) Foley et al. 2012a; (13) Gutiérrez et al. 2011; (14) Richmond \& Smith 2012; (15) CfA Supernova Group, unpublished (http://www.cfa.harvard.edu/supernova/sn12cg.html); (16) Frogel et al. 1987; (17) Richmond et al. 1995; (18) Matheson et al. 2012.

selected since, among the DIBs, it offers an optimal combination of strength and narrow line width. Upper limits $(3 \sigma)$ were calculated from signal-to-noise ratio measurements using an empirical relationship derived from the error estimates returned by fitprofs for those objects where the $5780 \AA$ feature was detected. In the case of SN 2009ig, a high signal-to-noise ratio spectrum obtained with UVES on the ESO Very Large Telescope was used to calculate a tighter upper limit on the equivalent width of the host $5780 \AA$ absorption than allowed by our MIKE data.

Total column densities for the 46 objects in our Milky Way sample are given in Table 1 . Total column density and equivalent width measurements for the $32 \mathrm{SNe}$ Ia in the host absorption sample are found in Table 3. Figure 1 shows the VPFIT host absorption profile fits for the SNe where both the $\mathrm{Na}$ I D and K I lines were detected, while Figure 2 shows the fits of the host $\mathrm{Na}$ I D lines for those objects for which the $\mathrm{K}_{\mathrm{I}}$ lines were not observed or detected.

\subsection{Dust Extinction}

We adopt the Schlafly \& Finkbeiner (2011) re-calibration of the Schlegel et al. (1998) extinction map to estimate the Milky Way component of the visual extinction, $A_{V}$, for the 
Table 3

SNe Ia Host Absorption-line Measurements

\begin{tabular}{|c|c|c|c|c|c|c|}
\hline $\begin{array}{l}\mathrm{SN} \\
\text { (1) }\end{array}$ & $\begin{array}{c}\text { Sternberg } \\
\text { Type } \\
(2)\end{array}$ & $\begin{array}{l}\text { Reference } \\
\text { (3) }\end{array}$ & $\begin{array}{c}\lambda / \Delta \lambda \\
(4)\end{array}$ & $\begin{array}{c}\log N_{\mathrm{NaI}} \\
\left(\mathrm{cm}^{-2}\right) \\
(5)\end{array}$ & $\begin{array}{c}\log N_{\mathrm{K}_{\mathrm{I}}} \\
\left(\mathrm{cm}^{-2}\right) \\
(6)\end{array}$ & $\begin{array}{c}\mathrm{EW}(5780) \\
(\mathrm{m} \AA) \\
(7)\end{array}$ \\
\hline $1986 \mathrm{G}$ & B & 1 & 20,000 & $13.740 \pm 0.050$ & $\cdots$ & $335 \pm 5$ \\
\hline 1994D & S & 2 & 38,000 & $11.800 \pm 0.020$ & $\cdots$ & $\cdots$ \\
\hline $2001 \mathrm{cp}$ & $\mathrm{R}$ & 3 & 30,000 & $12.970 \pm 0.470$ & $\cdots$ & $\ldots$ \\
\hline 2001da & $\mathrm{R}$ & 3 & 30,000 & $12.512 \pm 0.073$ & $\ldots$ & $\ldots$ \\
\hline 2001el & $\mathrm{R}$ & 4 & 50,000 & $12.76 \pm 0.03$ & $11.29 \pm 0.03$ & $189 \pm 3$ \\
\hline 2002bo & B & 3 & 30,000 & $14.406 \pm 0.862$ & $\cdots$ & $37 \pm 7$ \\
\hline $2002 \mathrm{cr}$ & $S$ & 3 & 30,000 & $12.329 \pm 0.088$ & $\cdots$ & $<47$ \\
\hline 2002ha & B & 3 & 30,000 & $12.886 \pm 0.125$ & $\ldots$ & $\ldots$ \\
\hline 2002jg & B & 3 & 30,000 & $13.246 \pm 0.021$ & $\ldots$ & $\ldots$ \\
\hline $2006 X$ & B & 5 & 48,000 & $13.779 \pm 0.041$ & $11.995 \pm 0.035$ & $<72$ \\
\hline $2006 \mathrm{~cm}$ & B & 5 & 48,000 & $15.242 \pm 0.069$ & $12.307 \pm 0.099$ & $441 \pm 20$ \\
\hline 2007af & $\mathrm{R}$ & 5 & 48,000 & $12.044 \pm 0.142$ & $\ldots$ & $<37$ \\
\hline $2007 \mathrm{fb}$ & B & 5 & 48,000 & $12.844 \pm 0.024$ & $\ldots$ & $\ldots$ \\
\hline $2007 \mathrm{fs}$ & S & 5 & 48,000 & $12.583 \pm 0.029$ & $\cdots$ & $<31$ \\
\hline 2007hj & $\cdots$ & 5 & 52,000 & $<12.05$ & $\ldots$ & $\ldots$ \\
\hline $2007 \mathrm{kk}$ & $\mathrm{B}$ & 5 & 52,000 & $12.595 \pm 0.171$ & $\ldots$ & $\ldots$ \\
\hline 2007le & B & 5 & 52,000 & $13.281 \pm 0.012$ & $<11.14$ & $139 \pm 4$ \\
\hline 2007on & $\cdots$ & 5 & 48,000 & $<11.32$ & $\cdots$ & $\cdots$ \\
\hline $2007 \mathrm{sr}$ & $S$ & 5 & 25,000 & $13.220 \pm 0.182$ & $11.241 \pm 0.109$ & $71 \pm 4$ \\
\hline $2008 \mathrm{C}$ & B & 5 & 48,000 & $12.720 \pm 0.184$ & $\ldots$ & $<43$ \\
\hline $2008 \mathrm{ec}$ & B & 5 & 54,000 & $13.055 \pm 0.044$ & $\cdots$ & $77 \pm 7$ \\
\hline $2008 \mathrm{fp}$ & $\mathrm{B}$ & 5 & 35,000 & $14.472 \pm 0.036$ & $12.056 \pm 0.033$ & $86 \pm 11$ \\
\hline $2008 \mathrm{hv}$ & $\cdots$ & 5 & 35,000 & $<11.18$ & $\cdots$ & $<25$ \\
\hline 2008 ia & $\cdots$ & 5 & 35,500 & $<11.39$ & $\cdots$ & $\cdots$ \\
\hline SNF20080514-002 & $\cdots$ & 5 & 52,000 & $<11.65$ & $\ldots$ & $\ldots$ \\
\hline $2009 \mathrm{ds}$ & $\mathrm{B}$ & 5 & 35,500 & $12.920 \pm 0.058$ & $<10.93$ & $\cdots$ \\
\hline 2009ig & B & 5 & 34,500 & $13.105 \pm 0.161$ & $11.247 \pm 0.034$ & $<18$ \\
\hline 2009le & $\mathrm{R}$ & 5 & 35,500 & $13.254 \pm 0.103$ & $11.118 \pm 0.073$ & $<25$ \\
\hline $2010 \mathrm{~A}$ & $\mathrm{R}$ & 5 & 35,500 & $12.605 \pm 0.021$ & $<11.11$ & $99 \pm 10$ \\
\hline $2010 \mathrm{ev}$ & S & 5 & 35,500 & $12.701 \pm 0.029$ & $10.949 \pm 0.085$ & $227 \pm 10$ \\
\hline $2011 \mathrm{fe}$ & $S$ & 6 & 82,000 & $11.495 \pm 0.024$ & $\ldots$ & $\cdots$ \\
\hline $2012 \mathrm{cg}$ & $\mathrm{R}$ & 7 & 47,000 & $12.989 \pm 0.035$ & $11.255 \pm 0.075$ & $84 \pm 5$ \\
\hline
\end{tabular}

Notes. Columns: (1) SN name; (2) Classification of Na I D profile as per Sternberg et al. (2011) (B= Blueshifted, R= Redshifted, $\mathrm{S}=$ Single/Symmetric); (3) High-dispersion spectroscopy reference; (4) Spectral resolution; (5) Logarithm of the total neutral sodium column density; (6) Logarithm of the total neutral potassium column density; (7) Equivalent width of DIB feature at $5780 \AA$. .

References. (1) D’Odorico et al. 1989; (2) Ho \& Filippenko 1995; (3) Foley et al. 2012b; (4) Sollerman et al. 2005; (5) Sternberg et al. 2011; (6) Patat et al. 2013; (7) S. Raskutti et al. 2013, in preparation.

objects in both our Milky Way and host absorption samples. Measuring the dust extinction produced in the SN host galaxy is a more complicated matter. In general, SNe Ia in low-reddening environments display intrinsic colors at maximum light that vary uniformly with the decline rate parameter, $\Delta m_{15}(B)$, and these relations can be used to derive color excesses such as $E(B-V)$. Fundamentally, however, it is $A_{V}$ that is proportional to the column density of the dust, and converting from $E(B-V)$ to $A_{V}$ requires knowledge of the ratio of total-to-selective extinction, $R_{V}$. In the Milky Way, the distribution of $R_{V}$ values is strongly peaked at $\sim 3$ (e.g., see Fitzpatrick \& Massa 2007), whereas the situation is not so clear for $\mathrm{SNe}$ Ia in other galaxies. Cosmological studies solving for an average value of $R_{V}$ by minimizing the Hubble diagram dispersion inevitably yield values $\lesssim 2$ (e.g., Astier et al. 2006; Conley et al. 2007; Kessler et al. 2009), whereas ratios of color excesses at optical and NIR wavelengths for individual SNe Ia indicate that while most heavily-reddened events have $R_{V}<2$, the color excesses of many SNe Ia with $E(B-V)<0.3$ are consistent with the standard Galactic value of $R_{V} \sim 3$ (Folatelli et al. 2010; Mandel et al. 2011; Phillips 2012; C. Burns et al. 2013, in preparation).
In this study, we employ a Markov-Chain Monte Carlo (MCMC) code to simultaneously estimate most-likely values of $A_{V}$ and $R_{V}$ from optical and (when available) NIR light curves of the SNe Ia listed in Table 2. Sources for these data are given in Columns 4 and 5 of the table. Briefly, the code models the observed pseudo-colors ${ }^{27}$ of each SN as a combination of an intrinsic color dependent on the decline rate parameter $\Delta m_{15}(B)$ and a color excess due to dust extinction along the line-of-sight. The dust extinction, $A_{X}$, in any band, $X$, is modeled using the Cardelli et al. (1989) extinction law, which has two free parameters: $A_{V}$ and $R_{V}$. Given these two parameters and the observed value of $\Delta m_{15}(B)$, the observed pseudo-color at maximum, $m_{X}-m_{Y}$, can be computed as:

$m_{X}-m_{Y}=P_{X Y}^{2}\left(\Delta m_{15}(B)-1.1\right)+A_{X}\left(A_{V}, R_{V}\right)-A_{Y}\left(A_{V}, R_{V}\right)$

where $P_{X Y}^{2}$ is a second-degree polynomial that describes the intrinsic $m_{X}-m_{Y}$ color at maximum of $\mathrm{SNe}$ Ia (e.g., see

\footnotetext{
27 The pseudo-colors at maximum are computed by taking the difference of the maxima of each filter's light-curve.
} 
$\mathrm{Na}$ I D
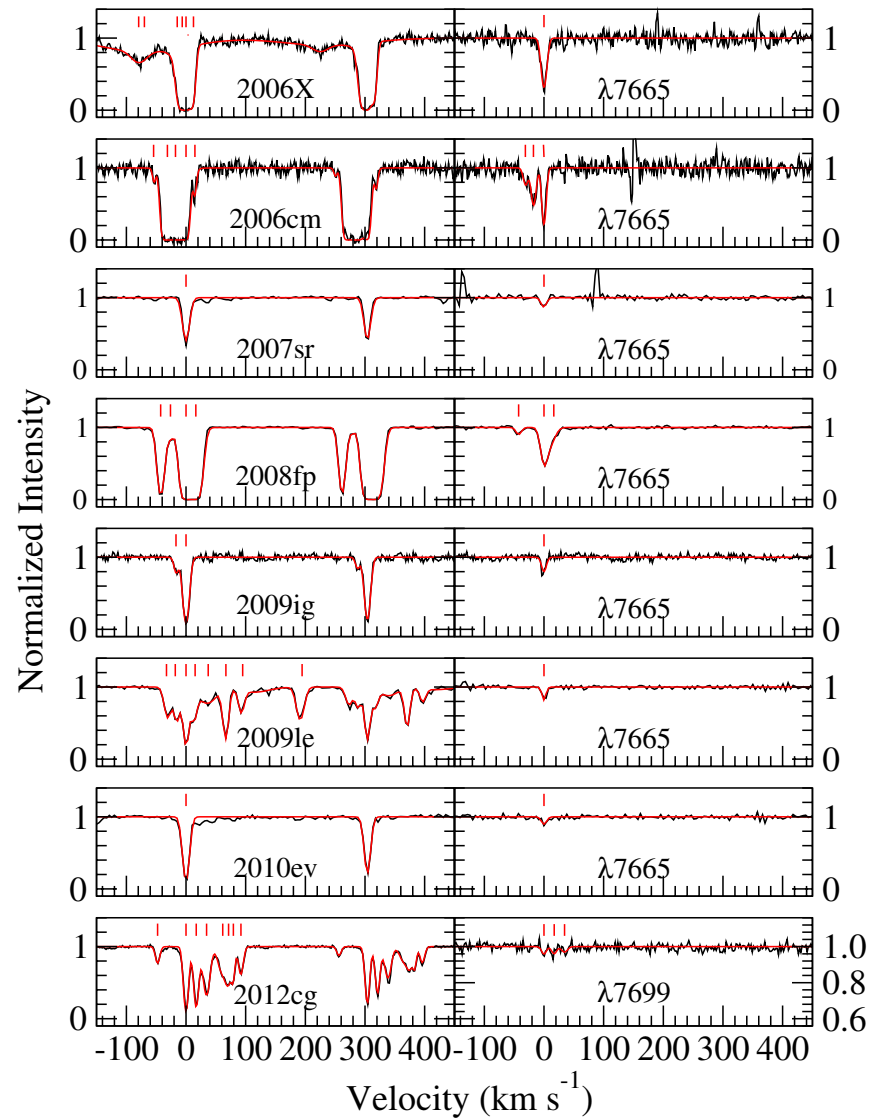

Figure 1. VPFIT models for the Na I D and K I absorption lines for those SNe Ia where both features were detected. The $\mathrm{Na}$ I $\mathrm{D}$ lines are shown in the left side of the figure. In the right half the $\mathrm{K}$ I absorption is displayed. For all but SN 2009le, the stronger component of the $\mathrm{K}_{\mathrm{I}}$ doublet, $\lambda 7665$, is plotted. In the case of SN 20091 e, the $\lambda 7665$ line is blended with strong telluric absorption, and so for this object the $\lambda 7699$ transition is shown. The red lines in each spectrum indicate the velocities of the components used to fit the profiles. The most prominent (deepest) component is used to set the zero point of the velocity scale.

(A color version of this figure is available in the online journal.)

Figure 2 of Phillips 2012). Note that nowhere do we need to know the distance to the SN Ia as we are dealing with colors only. We determine the pseudo-colors at maximum for both the training sample and the objects in this paper using SNooPy (Burns et al. 2011) fits to the observed light-curves. For $N$ filters, there are only $N-1$ independent colors and we model the set $B-X$, where $X=u g r i V Y J H$. Any other color can be derived as the sum or difference of two of these colors. However, the errors in the colors for a single object are correlated as they all contain the same $B$ magnitude. These correlated errors are accounted for in the MCMC code.

For objects that are significantly reddened $(E(B-V) \gtrsim 0.2)$ and have full optical and NIR coverage, the value of $R_{V}$ can be constrained quite well. However, as the reddening decreases, the value of $R_{V}$ cannot be constrained so readily, and if we do not use a more restrictive prior, we cannot get an upper bound for $A_{V}$ (a lower bound of zero is implicitly assumed). This is also the situation when we do not have NIR coverage. We therefore adopt a flat prior for $A_{V}$, while for $R_{V}$ a twoGaussian prior is employed consisting of a sharp component centered at $R_{V}=2.23$ that carries most of the distribution, plus a smaller $(\simeq 20 \%)$ contribution from a Gaussian centered at $R_{V}=3.27$ with very large spread. This prior is expressed

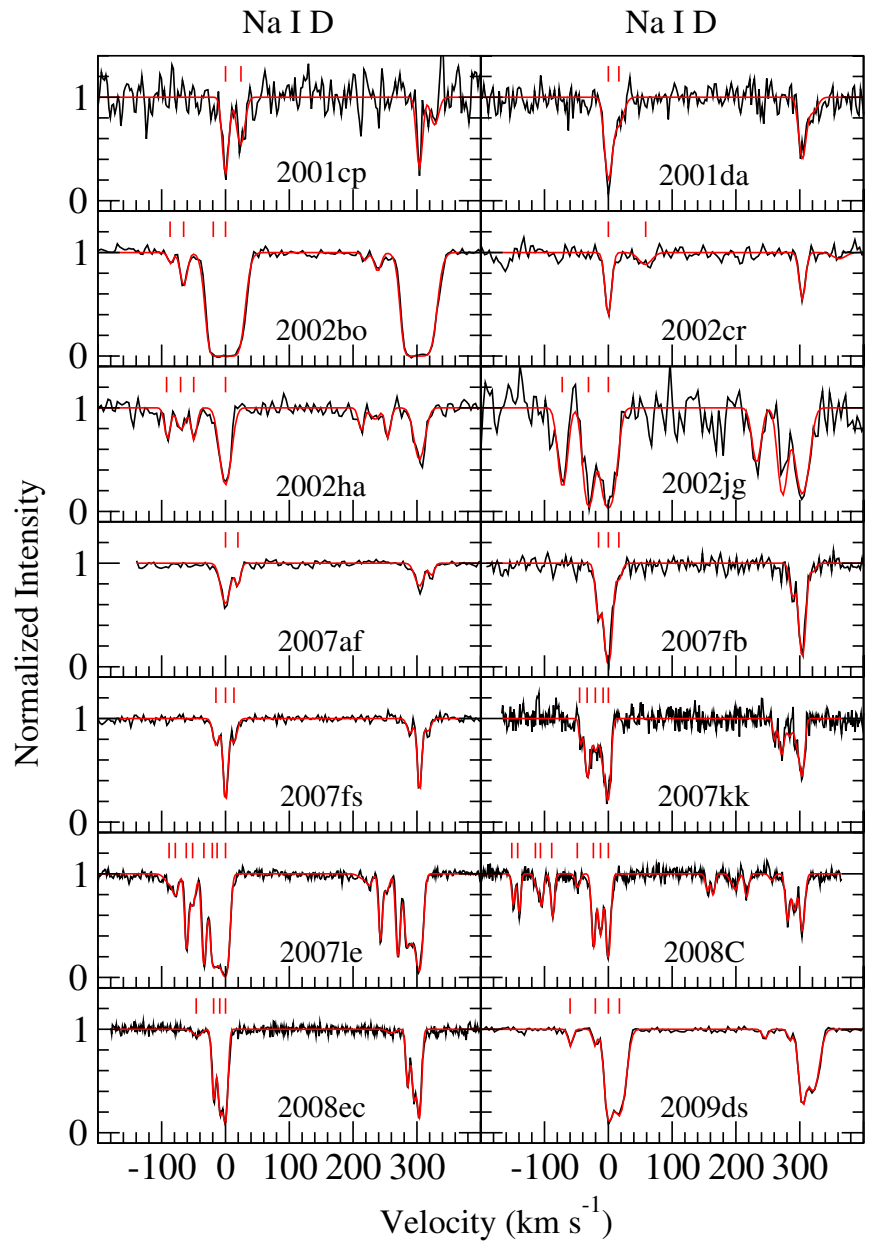

Figure 2. VPFIT models for the Na I D absorption lines for SNe Ia where the $\mathrm{K}$ I lines were not observed or detected. The red lines above the D2 line in each spectrum indicate the velocities of the components used to fit the profiles. The most prominent (deepest) component is used to set the zero point of the velocity scale.

(A color version of this figure is available in the online journal.)

mathematically as:

$$
\begin{aligned}
p\left(R_{V} \mid A, \mu_{1}, \sigma_{1}, \mu_{2}, \sigma_{2}\right) \sim & A \times e^{\left(-0.5 \times\left(R_{V}-\mu_{1}\right)^{2} / \sigma_{1}^{2}\right)}+(1-A) \\
& \times e^{\left(-0.5 \times\left(R_{V}-\mu_{2}\right)^{2} / \sigma_{2}^{2}\right)}
\end{aligned}
$$

where $A, \mu_{1}, \mu_{2}, \sigma_{1}$, and $\sigma_{2}$ are fixed at the following values derived from a training set of $74 \mathrm{SNe}$ Ia observed in the optical (ugriBV) by the Carnegie Supernova Project (CSP), 54 of which also had NIR $(Y J H)$ coverage (see C. Burns et al. 2013, in preparation, for further details):

$$
A=0.97 ; \quad \mu_{1}=2.23 ; \quad \sigma_{1}=0.4 ; \quad \mu_{2}=3.27 ; \quad \sigma_{2}=8 .
$$

The two-Gaussian prior was motivated by the observed distribution of values of $R_{V}$ derived from the CSP (Hamuy et al. 2006) sample of SNe Ia, which is strongly peaked at $R_{V} \sim 2$, but has long tails to larger and smaller values of $R_{V}$. One additional constraint on $R_{V}$ is that it be strictly positive.

For each color $m_{X}-m_{Y}$, we assume $P_{X Y}^{2}$ is the same for all $\mathrm{SNe}$ Ia, but that each SN has its own $A_{V}$ and $R_{V}$. Of course, $A_{V}$ and $R_{V}$ are highly covariant (indeed are completely degenerate for a single color). We therefore require several independent colors to properly constrain $A_{V}$ and $R_{V}$. Having NIR colors is of great help because the relative extinction becomes quite 

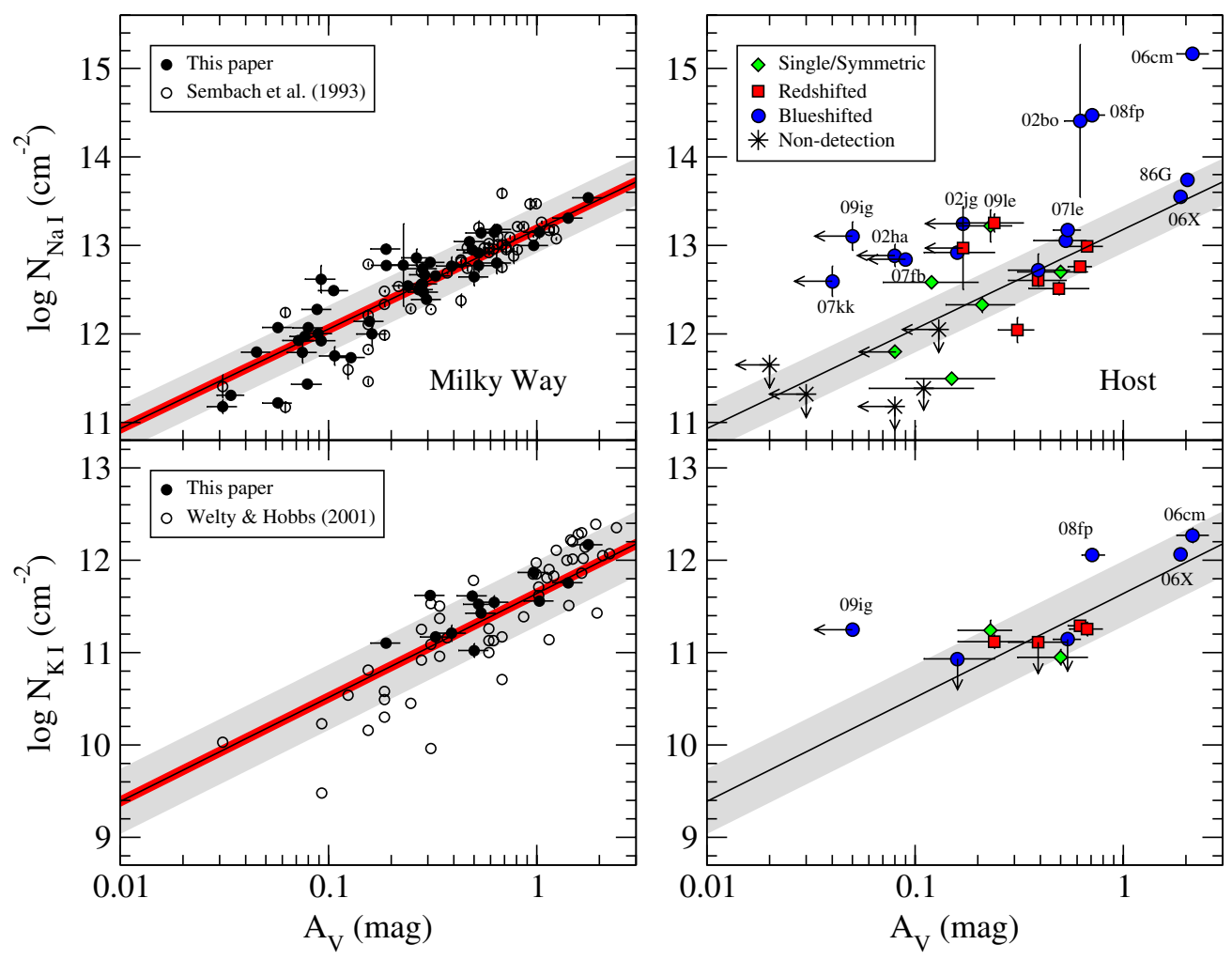

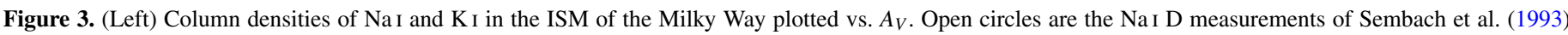

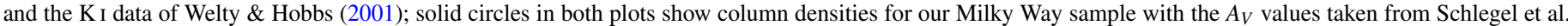

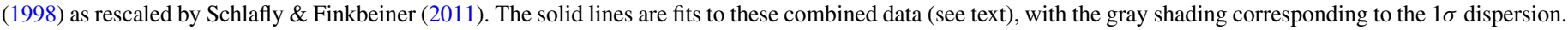

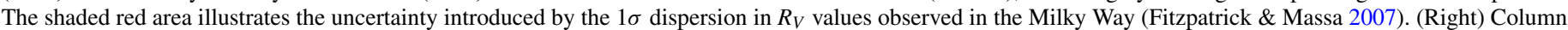

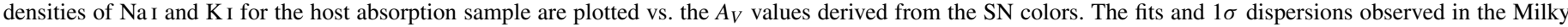

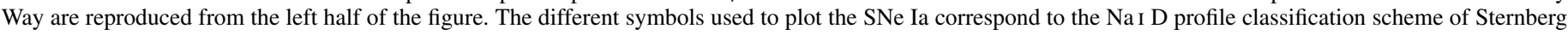
et al. (2011).

(A color version of this figure is available in the online journal.)

insensitive to $R_{V}$ at longer wavelengths, allowing us to break the $A_{V} / R_{V}$ degeneracy. In these cases, the two-Gaussian prior on $R_{V}$ has little effect on the derived value of $R_{V}$. However, when there is no NIR photometry or $E(B-V)$ is very small, the prior on $R_{V}$ limits the possible values of $A_{V}$.

Using MCMC techniques, we simultaneously solve for $R_{V}$ and $A_{V}$ for each $\mathrm{SN}$ using all independent colors available. We construct histograms for $R_{V}$ and $A_{V}$ by binning the Markov chains. We then compute the mode and $1 \sigma$ errors by bracketing $34 \%$ of the area to each side. The final calculated values are given in Columns 6 and 7 of Table 2. Where the posterior probability distribution is significantly non-symmetric, we report upper and lower bounds.

\section{RESULTS}

\subsection{Na I and $K_{I}$}

The upper-left panel of Figure 3 displays total Galactic Na I column densities for the 46 objects in our Milky Way sample plotted versus the $A_{V}$ values inferred from the Schlafly \& Finkbeiner (2011) Galactic reddenings. In calculating $A_{V}$, a value of $R_{V}=3.1$ is assumed. The red shaded area in Figure 3 illustrates the uncertainty in these $A_{V}$ values introduced by the $1 \sigma$ dispersion of \pm 0.27 in $R_{V}$ values observed in the Milky Way (Fitzpatrick \& Massa 2007). Shown for comparison are column density measurements obtained from profile fits to the $\mathrm{Na}$ I D lines by Sembach et al. (1993) for a sample of 50 distant $(d>1 \mathrm{kpc})$ late-O and early-B stars in low-density regions of the Milky Way disk and halo. A fit to the combined (Milky Way + Sembach et al. (1993)) sample of 96 measurements gives

$$
\log N_{\mathrm{Na}}=13.180(0.003)+1.125(0.005) \times \log A_{V},
$$

where the uncertainties in the slope and intercept are given in parentheses. This fit is plotted in the upper-left panel of Figure 3, with the gray shading indicating the $1 \sigma$ dispersion in $\log N_{\mathrm{Na}}$ of 0.26 dex.

In the lower-left panel of Figure 3, a similar plot of K I column densities versus $A_{V}$ is shown for the objects in our Milky Way sample for which a Galactic component of the $\mathrm{K}_{\mathrm{I}} \lambda \lambda 7665$, 7699 doublet was detected. The open circles are KI column densities derived from the $\mathrm{K}_{\mathrm{I}} \lambda 7699$ line for 52 stars in the Milky Way by Welty \& Hobbs (2001). The solid line is a fit to the combined data, with the gray shading indicating the $1 \sigma$ dispersion of 0.35 dex. Since an essentially linear relationship exists in the Milky Way between $\log N_{\mathrm{Na}}$ and $\log N_{\mathrm{K}}$ (Welty $\&$ Hobbs 2001), the slope of the fit was set equal to the slope of the $\log N_{\mathrm{Na}}$ versus $\log A_{V}$ relation in the upper-left panel of Figure 3, yielding

$$
\log N_{\mathrm{KI}}=11.639(0.005)+1.125 \times \log A_{V} .
$$

Note that the dispersions in the stellar relations are large, and if one were to use the fits to estimate $A_{V}$ from a measurement of the $\mathrm{Na}$ I or K I column density, the error would be $54 \%$ or $72 \%$ of $A_{V}$ itself, respectively. 


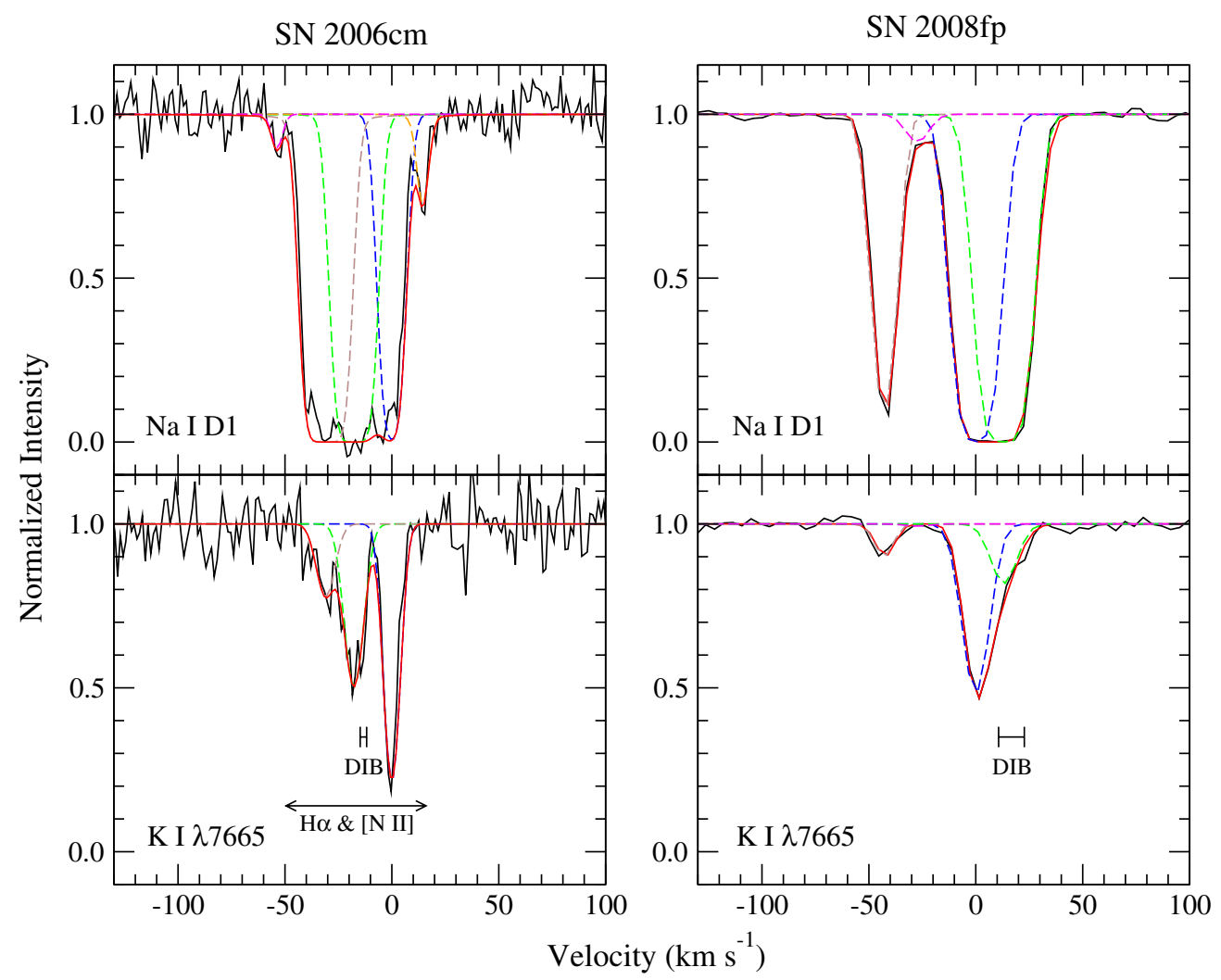

Figure 4. Host Na I D1 and $\mathrm{K}_{\mathrm{I}} \lambda 7665$ absorption in SNe $2006 \mathrm{~cm}$ and 2008fp. The observations correspond to the black line. The best-fitting VPFIT model is shown as the red line, with the individual Voigt profile components plotted as dashed lines. Zero velocity has been arbitrarily set to correspond to the strongest component of the K I $\lambda 7665$ profile. The velocities of the $5780 \AA$ DIB in each SN are shown as $\pm 1 \sigma$ error bars, and were derived assuming a Gaussian profile of $2.1 \AA$ FWHM and a rest wavelength of $5780.55 \AA$ (Tuairisg et al. 2000). The FWHM velocity range covered by the $\mathrm{H} \alpha$ and [N II] emission in the extracted spectrum of SN 2006 $\mathrm{cm}$ is also indicated.

(A color version of this figure is available in the online journal.)

The right half of Figure 3 shows the same plots, but this time for the host absorption components of the $\mathrm{Na}$ I and $\mathrm{K}$ I lines. The fits and $1 \sigma$ dispersions corresponding to the Milky Way relations are reproduced from the left half of Figure 3 for comparison. The symbols used to plot the SNe Ia measurements follow the classification scheme based on the structure of the Na I D profile employed by Sternberg et al. (2011), with the three categories defined as follows:

1. Blueshifted: one prominent absorption feature with weaker features at shorter wavelengths with respect to it.

2. Redshifted: one prominent absorption feature with weaker features at longer wavelengths with respect to it.

3. Single/Symmetric: a single absorption feature, or several features with both blue and redshifted structures of similar magnitude.

Figure 3 shows that only 1 of 96 objects in the Milky Way + Sembach et al. (1993) sample has a Na I column density that lies $>3 \sigma$ from the fit defined by Equation (4), consistent with the statistical expectation. However, 8 of $27(30 \%)$ of the SNe Ia host galaxy Na I column densities fall $>3 \sigma$ above the Galactic fit. Even more striking is the fact that all eight of these deviant $\mathrm{SNe}$ Ia have "Blueshifted" host Na I D absorption profiles. Some of these (e.g., SNe 2007kk and 2009ig) are objects with significant Na I column densities, but for which the optical and NIR colors imply quite small amounts of dust extinction. Note that one of the "Blueshifted" SNe, $2006 \mathrm{~cm}$, was originally classified by Sternberg et al. (2011) as "Single/Symmetric." The Na I D1 and $\mathrm{K}_{\mathrm{I}} \lambda 7665$ profiles for this SN are displayed in Figure 4. The
D1 line is highly saturated making it difficult to categorize, but the much weaker KI $\lambda 7665$ absorption reveals that SN $2006 \mathrm{~cm}$ should actually be classified as "Blueshifted."

In our sample, there are 14 "Blueshifted," 7 "Redshifted," and 6 "Single/Symmetric" SNe. The Sternberg et al. (2011) sample had very similar numbers-15, 6, and $6 \mathrm{SNe}$, respectively, in these three categories - and so our sample should be representative of theirs. The probability in a random draw that all eight of the SNe Ia in Figure 3 lying more than $3 \sigma$ from the Milky Way relationship would belong to the "Blueshifted" class is $0.14 \%$ and, therefore, highly unlikely. ${ }^{28}$

Five of the SNe Ia in our sample (2007hj, 2007on, 2008hv, 2008ia, and SNF20080514-002) did not show detectable host $\mathrm{Na}$ I D absorption in their spectra. The optical and NIR colors of these objects, all of which occurred in E or S0 host galaxies, identify them as having suffered little or no dust reddening, consistent with the absence of detectable Na I D lines. Upper limits $(3 \sigma)$ on $\log N_{\mathrm{Na}}$ for these non-detections are given in Table 3 and plotted in Figure 3.

Due to the weakness of the KI $\lambda \lambda 7665,7699$ doublet, we were able to measure host $\mathrm{K}_{\mathrm{I}}$ column densities for only eight of the $\mathrm{SNe}$ Ia in our host absorption sample. Upper limits $(3 \sigma)$ were measured for an additional three $\mathrm{SNe}$ with high signal-to-noise ratio spectra. These measurements, along

\footnotetext{
28 It might be argued that the "Single/Symmetric" SN 1994D and the "Redshifted" SN 2001cp, for which we are only able to place an upper limit on $A_{V}$, should also be included in this calculation. Doing so increases the probability of a chance occurrence to $\sim 1.5 \%$.
} 

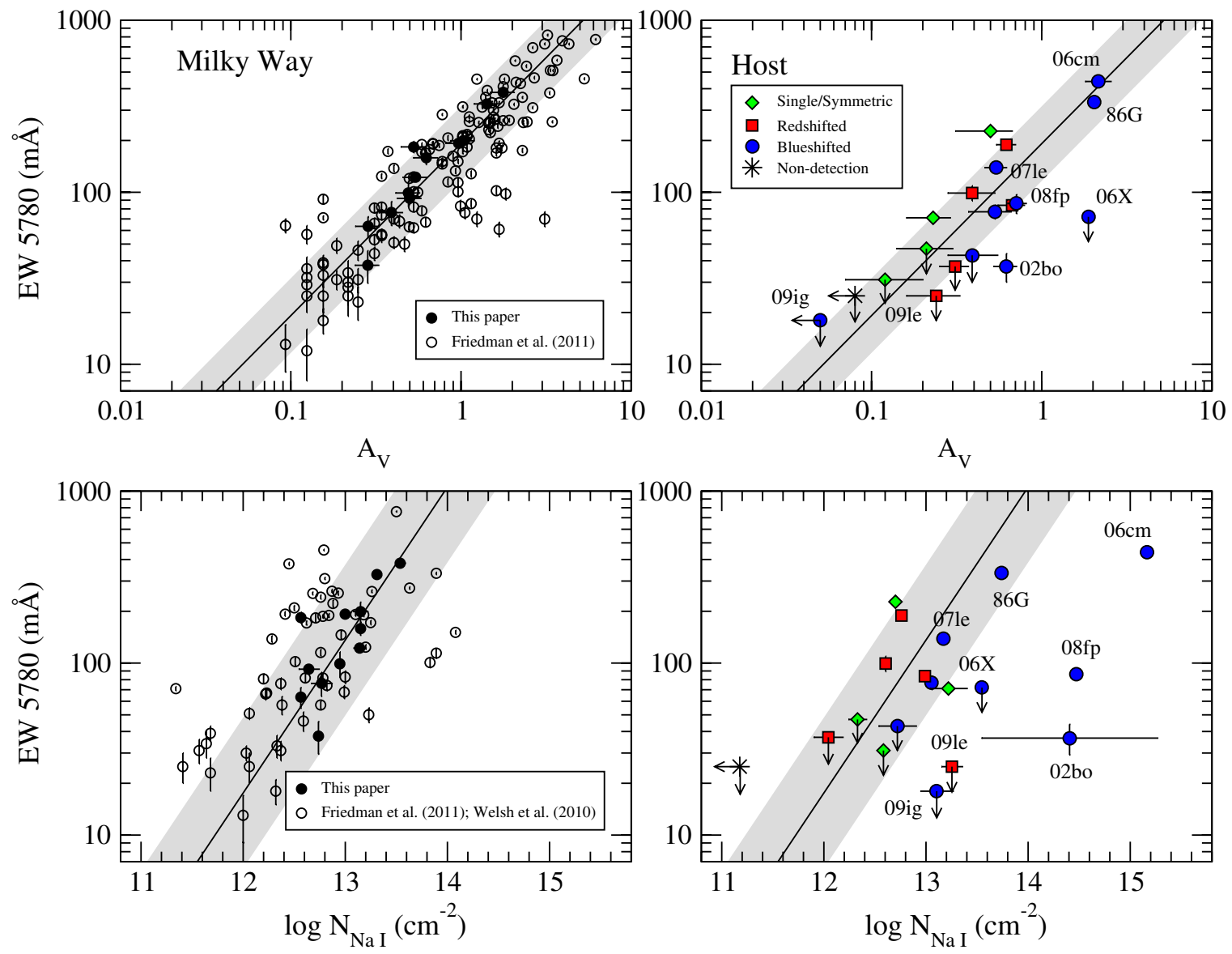

Figure 5. (Left) Equivalent width of the DIB feature at $5780 \AA$ in the ISM of the Milky Way plotted vs. $A_{V}$ and the column density of Na I. The open circles in the upper plot correspond to the measurements of Friedman et al. (2011); those in the lower plot are a combination of the EW $5780 \AA$ values of Friedman et al. (2011) with Na I column densities from Welsh et al. (2010). The solid circles in both plots correspond to the objects in our Milky Way sample for which a Galactic component of the $5780 \AA$ feature was detected. The $A_{V}$ values for the latter objects are taken from Schlegel et al. (1998) as rescaled by Schlafly \& Finkbeiner (2011). The solid lines are fits to these combined data, with the gray shading corresponding to the $1 \sigma$ dispersions (see text). (Right) Equivalent width of the host DIB absorption at $5780 \AA$ plotted vs. $A_{V}$ values derived from the SN colors (upper) and the column density of Na I (lower). The fits and $1 \sigma$ dispersions observed in the Milky Way are reproduced from the left half of the figure. The different symbols used to plot the SNe Ia correspond to the Na I D profile classification scheme of Sternberg et al. (2011).

(A color version of this figure is available in the online journal.)

with the host KI column density for SN 2001el published by Sollerman et al. (2005), are given in Table 3 and plotted in the lower-right panel of Figure 3. Interestingly, of the three "Blueshifted" objects that lie more than $5 \sigma$ above the Galactic $N_{\mathrm{Na}}$ versus $A_{V}$ relationship-SNe $2006 \mathrm{~cm}, 2008 \mathrm{fp}$ and 2009ig-only SN 2009ig falls significantly above the corresponding $N_{\mathrm{K}}$ versus $A_{V}$ relationship.

\subsection{DIB at $5780 \AA$}

Although the carriers of DIBs remain unknown (Sarre 2006), they are ubiquitously present in the ISM of the Milky Way and beyond-from the Magellanic Clouds, M31, and M33, to starburst galaxies, SNe host galaxies and even damped Ly $\alpha$ systems (e.g., Cox et al. 2008; Heckman \& Lehnert 2000; Sollerman et al. 2005; York et al. 2006). The upper-left panel of Figure 5 displays the equivalent width of the DIB at $5780 \AA$ plotted as a function of $A_{V}$ for 131 late-O and early-B stars in the Milky Way (Friedman et al. 2011) (open circles), and for the 12 objects in our Milky Way sample for which we were able to detect a Galactic component of the $5780 \AA$ DIB absorption feature (solid circles). Previous studies (e.g., Herbig 1993; Welty et al. 2006; Friedman et al. 2011; Vos et al. 2011; Yuan \& Liu 2012) have found that the strength of the $5780 \AA$ feature is essentially linearly proportional to the amount of reddening, and so we assume this in deriving the following fit to these combined data:

$$
\log \operatorname{EW}(5780)=2.283(0.001)+\log A_{V} .
$$

The black line in the upper-left panel of Figure 5 shows this fit, which is in excellent agreement with that recently obtained by Yuan \& Liu (2012) for a much larger sample of stars. The gray shading indicates the $1 \sigma$ dispersion of 0.22 dex that translates to a $50 \%$ error in $A_{V}$ if the $5780 \AA$ feature is used to estimate the dust extinction for any single object.

As shown in the lower-left panel of Figure 5, the strength of the $5780 \AA$ feature also correlates reasonably well with the column density of Na I in the Milky Way (Herbig 1993; Welty et al. 2006). The open circles are DIB $5780 \AA$ equivalent widths for 55 early-type stars from Friedman et al. (2011) combined with Na I column densities measured from the D lines for the same stars by Welsh et al. (2010); the solid circles in the figure correspond to the measurements of the Galactic $5780 \AA$ A feature in our Milky Way sample. Equations (4) and (6) yield

$$
\log \mathrm{EW}(5780)=-9.433(0.004)+0.889(0.004) \times \log N_{\mathrm{NaI}},
$$

which provides a reasonable fit to the observations, albeit with a large dispersion of 0.44 dex. 


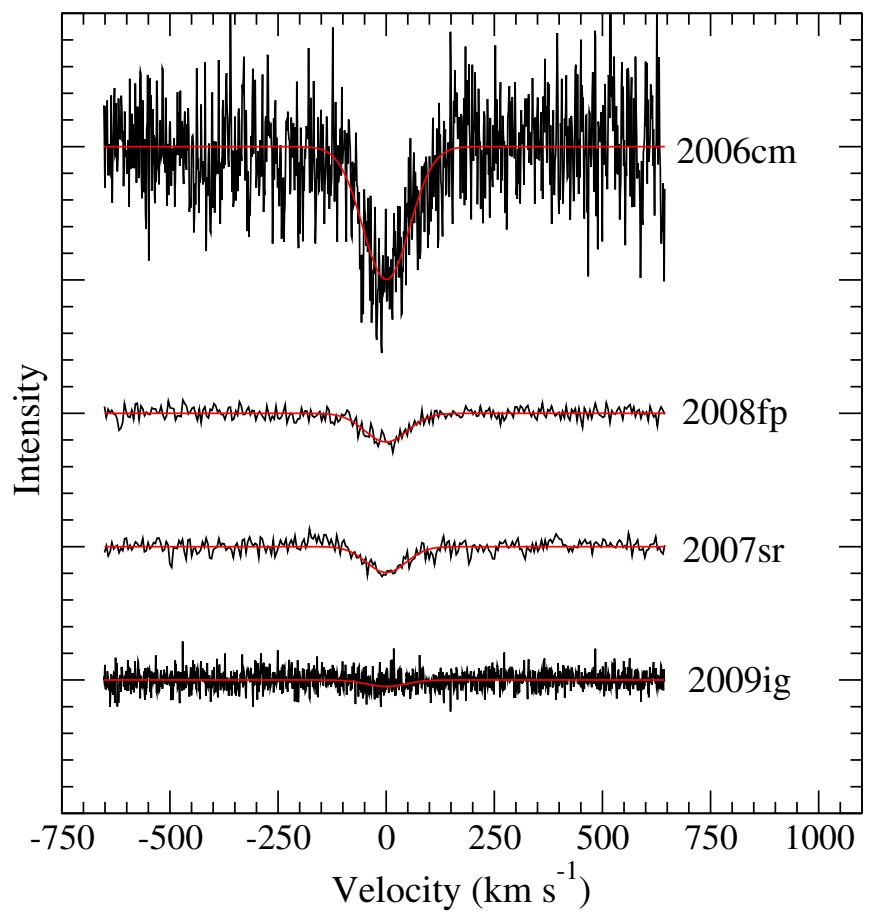

Figure 6. Examples of three detections of host DIB absorption at $5780 \AA$ (SNe $2006 \mathrm{~cm}, 2008 \mathrm{fp}$, and 2007sr). Spectral observations are plotted in black, and fits assuming a Gaussian profile of $2.1 \AA$ FWHM are in red. Also shown is the spectrum of SN 2009ig where the $5780 \AA$ is not clearly detected, but where the signal-to-noise ratio of the spectrum allowed a meaningful upper limit to be measured. The fit in this case corresponds to the derived $3 \sigma$ upper limit on the equivalent width. The scaling of the intensity axis is the same for each $\mathrm{SN}$ in order to preserve the relative differences in strength of the $5780 \AA$ feature; the spectra have been offset for illustration purposes.

(A color version of this figure is available in the online journal.)

The right half of Figure 5 shows the same plots for the host absorption component of the DIB at $5780 \AA$. This feature was detected in $34 \%$ (11 of 32) of the SNe Ia in our sample. Upper limits $(3 \sigma)$ are plotted for an additional eight objects. Figure 6 illustrates three examples of detections (SNe $2006 \mathrm{~cm}$, 2008fp, and 2007sr), and one where only an upper limit could be measured (SN 2009ig). The upper-right panel of Figure 5 indicates that in all cases where the $5780 \AA$ A feature was detected, its strength is compatible with the dust extinction implied by the SN colors. In general, the upper limits are also consistent with the Milky Way relation, with the exception of SN 2006X for which Cox \& Patat (2008) found the DIBs at $6196 \AA$ and $6283 \AA$ to be 2.5-3.5 times weaker than expected. Unfortunately, the echelle spectra obtained by these authors did not cover the $5780 \AA$ feature, and the signal-to-noise ratio of our own observation does not allow a tight upper limit to be measured. Based on the relations given by Friedman et al. (2011), the Cox $\&$ Patat (2008) measurements of the equivalent widths of the $6196 \AA$ and $6283 \AA$ features imply EW $5780 \AA=50-160 \mathrm{~m} \AA$, consistent with our upper limit.

The lower-right panel in Figure 5 shows that the correlation between the strength of the $5780 \AA$ feature and the Na I column density is particularly poor for the "Blueshifted" SNe Ia. As in Figure 3, SNe 2002bo, 2006cm, and 2008fp, and 2009ig have values of $N_{\mathrm{Na}}$ that are an order of magnitude or more greater than predicted by the Milky Way relationship. While the discrepancy of these four SNe Ia in Figure 3 could perhaps be attributed to large underestimates of $A_{V}$ from the SN colors, the fact that errors in $A_{V}$ have no effect on the points in the lower-right panel of Figure 5 argues against this explanation. We conclude, therefore, that the anomalously large Na I column densities in these SNe Ia is a real effect.

\section{DISCUSSION}

The observations presented in Section 3 can be summarized as follows:

1. One-fourth of the SNe Ia in our sample (8 of 32) exhibit host Na I D absorption that is more than $3 \sigma$ stronger than expected based on the amount of dust extinction implied by the $\mathrm{SN}$ colors and the Galactic relationship between $N_{\mathrm{Na}}$ and $A_{V}$. All of these SNe Ia with anomalously large Na I column densities belong to the "Blueshifted" class of Sternberg et al. (2011).

2. The relationship between the host $\mathrm{K}_{\mathrm{I}}$ absorption column densities and $A_{V}$ for the SNe Ia appear to be more consistent with the Milky Way relationship. While the number of detections is small and this result therefore requires verification, it is interesting to note that two of the "Blueshifted" objects with highly-discrepant Na I column densities, SNe $2006 \mathrm{~cm}$ and $2008 \mathrm{fp}$, showed essentially normal K I absorption for the dust extinction implied by their colors.

3. When detected in SNe Ia, the strength of the host absorption DIB $5780 \AA$ feature is consistent with the amount of dust extinction implied by the SN colors. Notably, this statement applies to the "Blueshifted" SNe 2002bo, 2006cm, 2008fp, and 2009ig, all of which displayed anomalously large Na I column densities.

Figure 7 illustrates just how unusually strong the Na I D absorption is for two SNe with "Blueshifted" host absorption profiles. In the upper-left panel, the Milky Way component of the Na I D lines in the spectrum of SN 2009le is plotted. The Galactic extinction for this object is $A_{V}=0.05 \pm 0.01$ mag. In the upper-right panel, the host absorption component of the D lines in the spectrum of the "Blueshifted" SN 2009ig is shown for comparison. Analysis of the light curves of SN 2009ig yields an upper limit for the host extinction of $A_{V}<0.05 \mathrm{mag}$, yet the D lines are clearly much stronger than the Milky Way absorption in SN 2009le. The lower half of Figure 7 shows a similar comparison of the Milky Way component of the Na I absorption in the spectrum of SN 2011ek [ $A_{V}$ (Galactic) $=$ $0.97 \pm 0.15 \mathrm{mag}]$, which is contrasted with the host absorption component of the D lines in the spectrum of the "Blueshifted" SN 2008fp $\left[A_{V}\right.$ (Host) $\left.=0.71_{-0.08}^{+0.10} \mathrm{mag}\right]$. Note the strongly saturated line profiles in the spectrum of the latter object, whereas the $\mathrm{Na}$ I lines produced by the interstellar medium of the Milky Way in SN 2011ek are considerably weaker despite the extinction being nominally somewhat greater. These two comparisons demonstrate visually that the anomalous strength of the host $\mathrm{NaI} \mathrm{D}$ absorption in these two $\mathrm{SNe}$ is very real, and not due to inaccuracies in measuring column densities of saturated lines.

\subsection{Na I, KI, and DIBs in the Local Group and Beyond}

In order to understand if systematic differences in the sodium and potassium abundances might occur due to potential metallicity effects in supernova host galaxies, we can examine similar trends for the Large and Small Magellanic Clouds (LMC and SMC) - two nearby galaxies which have significantly lower metallicities, by factors of two and five, respectively, compared to the Milky Way (Dufour 1984; Rolleston et al. 2002; Peimbert 


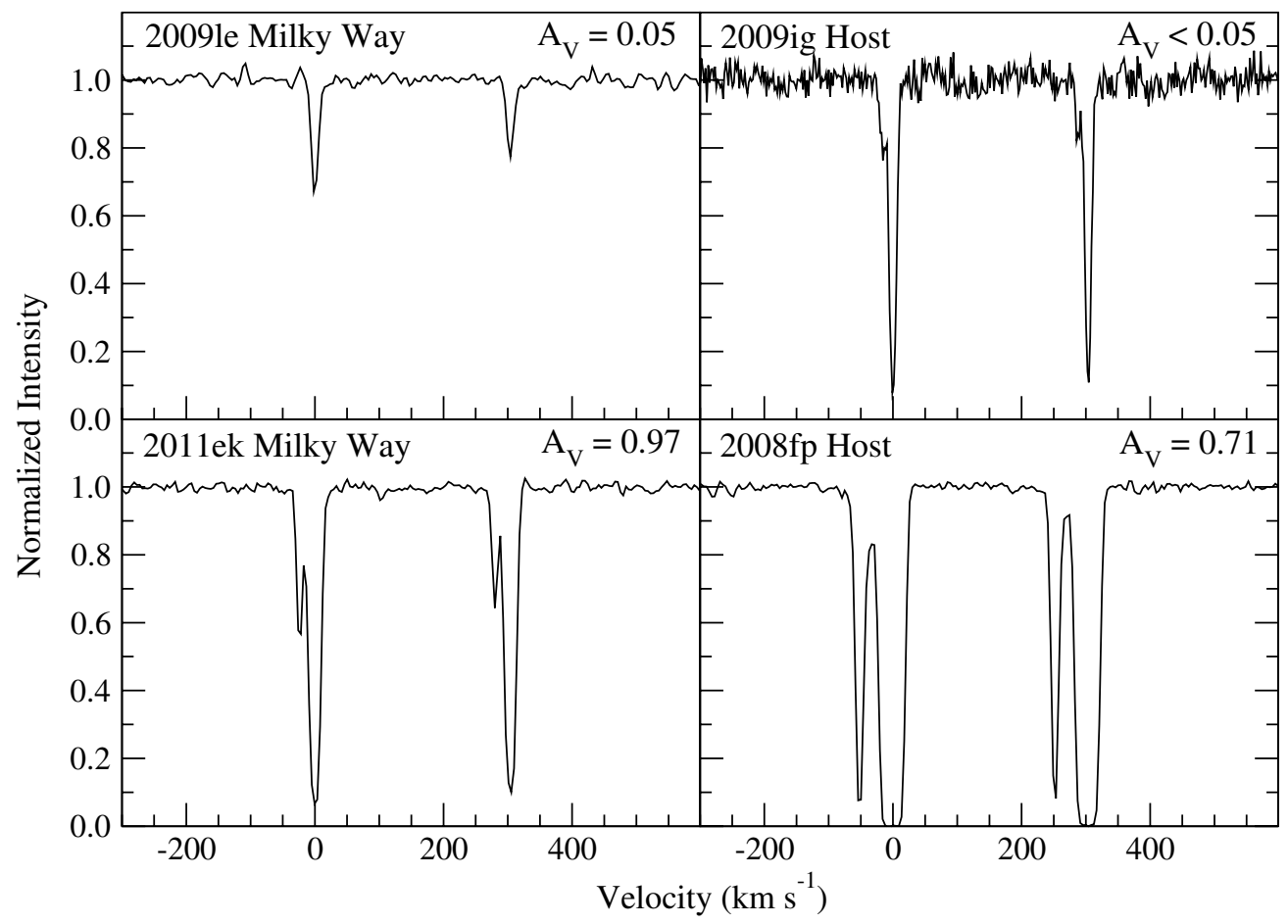

Figure 7. Na I D profiles of two of the objects in the Milky Way sample (left), and two SNe Ia in the host absorption sample with similar dust extinctions (right). Zero velocity has been arbitrarily set to correspond to the minimum of the D2 line absorption. The anomalous strength of the host Na I D absorption in SNe 2009ig and $2008 \mathrm{fp}$ is readily apparent.

\& Torres-Peimbert 1976; Bouret et al. 2003). The observed Na I and $\mathrm{K}$ I column densities of the diffuse ISM in both the LMC and SMC (Cox et al. 2006, 2007; Welty et al. 2006) are plotted as a function of $A_{V}$ in the left half of Figure 8. When only color excesses are provided by these authors, visual extinctions were computed using the average $R_{V}$ values given by Gordon et al. (2003). The measurements clearly reproduce the Galactic trends shown in Figure 3. Despite marked environmental differences such as the higher gas-to-dust ratio and stronger UV radiation field of the LMC, both $N_{\mathrm{Na}}$ and $N_{\mathrm{K}}$ follow the Galactic trend although the scatter in $N_{\mathrm{Na}}$ is somewhat larger. The $\mathrm{Na}$ I and $\mathrm{K}$ I column densities are also highly correlated in the Magellanic Clouds, with the ratio being similar to that found in the ISM of the Milky Way (see Section 4.5).

In this comparison it is important to understand that, due to the lower metallicity and stronger radiation fields, both $N_{\mathrm{Na}}$ and $N_{\mathrm{K}}$ are lower with respect to $N_{\mathrm{H}}$ in the Magellanic Clouds. However, the gas-to-dust ratio $N_{\mathrm{H}} / E(B-V)$, which scales approximately with metallicity, is also higher by a similar factor in the Magellanic Clouds (Welty et al. 2006). Both effects essentially cancel, resulting in similar correlations between $N_{\mathrm{Na}}, N_{\mathrm{KI}}$, and $A_{V}$ for both the Magellanic Clouds and the Milky Way. We conclude that it is unlikely that the enhanced $\mathrm{Na}$ I absorption observed for some $\mathrm{SNe}$ Ia is due to global host galaxy metallicity differences.

The panels on the right in Figure 8 display the observed $5780 \AA$ DIB equivalent width versus $A_{V}$ (top) and the Na I column density (bottom), respectively. In both cases, the equivalent width measurements of the $5780 \AA$ feature fall, on average, below the Galactic relation by a factor of $\sim 2$, consistent with the findings of Welty et al. (2006). As discussed by these authors, this is likely a consequence of lower metallicity, lower dust-togas ratios and molecular fractions, and the generally stronger radiation fields in the Magellanic Clouds. The similar behavior of the $5780 \AA$ DIB strength with respect to both $N_{\mathrm{Na}}$ and
$A_{V}$ is consistent with the good correlation between the latter two quantities (see the upper left panel of Figure 8). This is notably dissimilar to the situation for the $\mathrm{SNe}$ Ia, where the $5780 \AA$ A DIB strengths follow the Galactic $\mathrm{EW}(5780)$ versus $A_{V}$ relation, but do not follow the Galactic $\operatorname{EW}(5780)$ versus $N_{\mathrm{Na}}$ relation.

Unfortunately, recent determinations of $\mathrm{Na}$ I column densities for extra-Galactic sight-lines toward M31 and M33 suffer from saturation due to lower spectral resolution observations (Cordiner et al. 2011); accurate comparisons for these galaxies will require more sensitive high-resolution spectroscopic data. The measured $5780 \AA$ DIB strengths versus $E(B-V)$ in M31 are slightly above the Milky Way trend, but the sample is limited and prone to observational bias in preferentially detecting the strongest bands.

Beyond the Local Group, moderate-resolution observations of the NaI D lines and several DIBs, including the $5780 \AA$ feature, in starburst galaxies indicate that the equivalent widths follow the same dependence as Galactic DIBs on $E(B-V)$ and the Na I column density (Heckman \& Lehnert 2000). The $5780 \AA$ feature was detected by York et al. (2006) in the $z=0.5$ damped Ly $\alpha$ system of the QSO AO $0235+164$ and also found to be consistent with the Milky Way EW(5780) versus $E(B-V)$ relation. These authors also detected the Na I D2 line in this same system at an equivalent width of $\sim 0.8 \AA$. As discussed in the next section, equivalent widths are not as reliable as column densities for inferring dust extinction, but this value is not inconsistent with the reddening of $E(B-V)=0.23$ derived for the QSO (Junkkarinen et al. 2004) assuming the correlation observed in the Milky Way.

Sparks et al. (1997) detected extended interstellar Na I D absorption lines from gas associated with the compact emission filament system and dust lane in the central 20 arcsec of the dominant elliptical galaxy in the Centaurus cluster, NGC 4696. Due to the low resolution of their spectral observations, only 

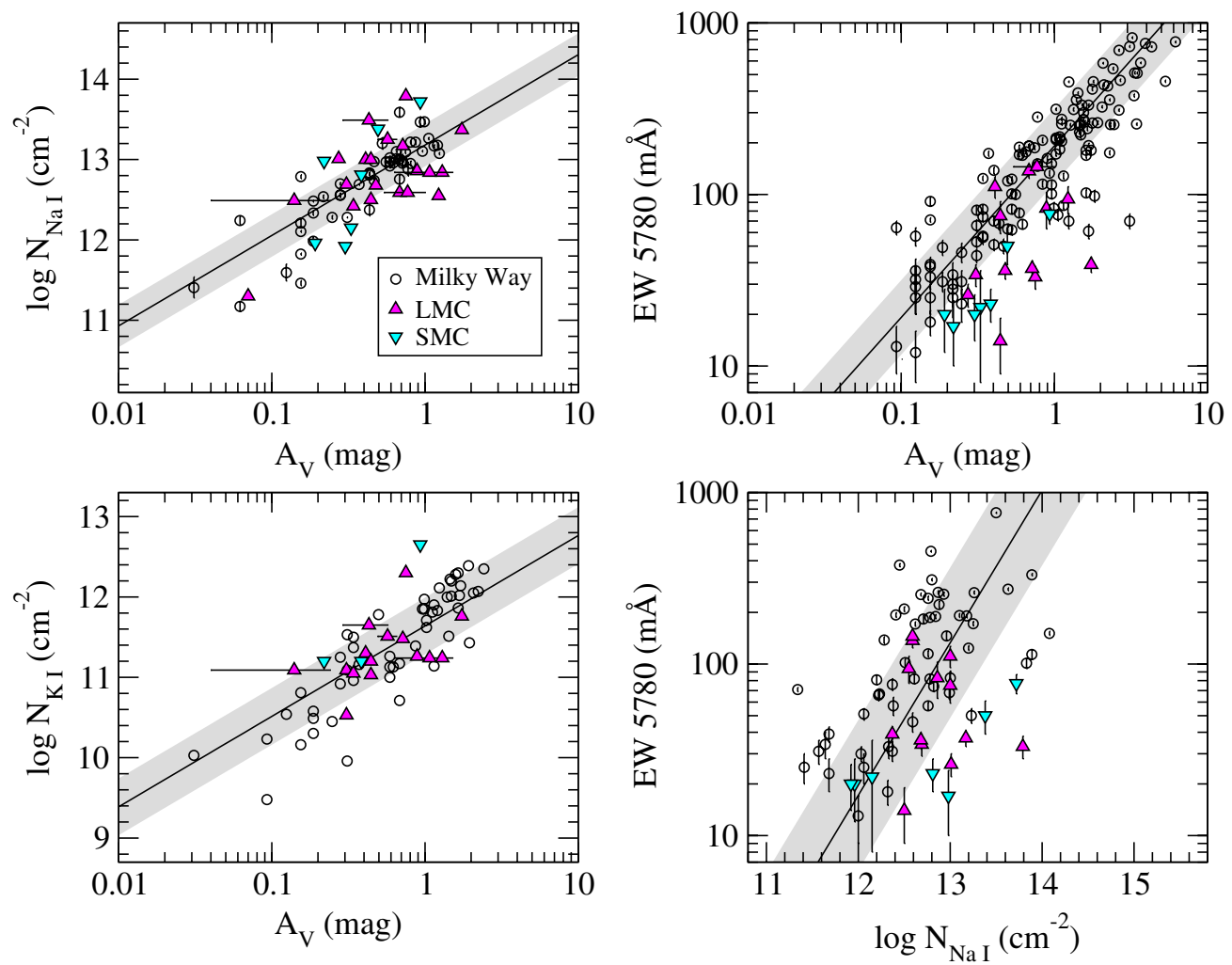

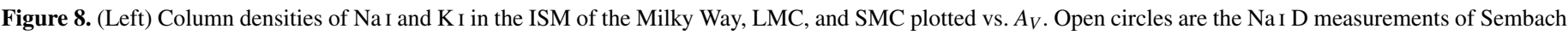

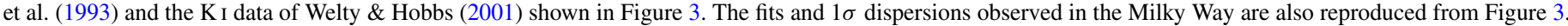

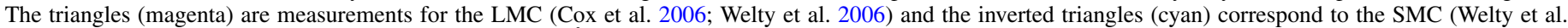

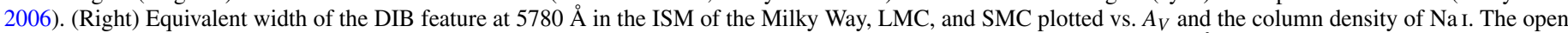

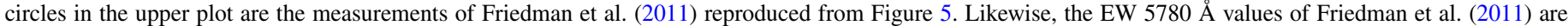

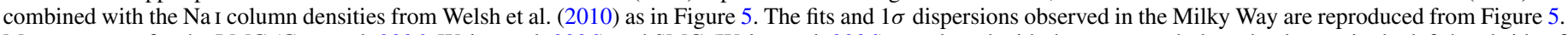

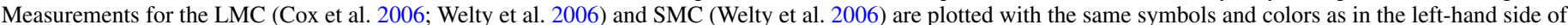
the figure.

(A color version of this figure is available in the online journal.)

total equivalent widths of the $\mathrm{Na}$ I absorption could be measured. Interestingly, the correlation between equivalent width and $E(B-V)$ was found to be significantly steeper than the standard Milky Way relation, suggesting a low dust-to-gas ratio. This is one of the few cases we are aware of in the literature where enhanced $\mathrm{Na}$ I absorption is observed in the ISM of a galaxy, although it should be emphasized that the environment is quite different from the disks of the spiral galaxy hosts of the majority of the SNe Ia in our sample.

\subsection{Equivalent Width of $\mathrm{Na}$ I $\mathrm{D}$ as an Indicator of Extinction}

The equivalent width of the Na I D lines, $\mathrm{EW}(\mathrm{Na}$ I D) is commonly measured in low-dispersion spectra to estimate the dust reddening for $\mathrm{SNe}$ Ia with poor photometric coverage, or peculiar SNe whose intrinsic colors are unknown (see Turatto et al. 2003, and references therein). It is interesting to compare the results for the $\mathrm{NaI} \mathrm{D}$ host absorption column densities presented in Section 3 with what equivalent widths give. This is shown in Figure 9 where the equivalent widths of the D lines measured from our echelle spectra are plotted versus $A_{V}$. Values for the Milky Way sample, augmented by high dispersion measurements of 82 stars (Sembach et al. 1993; Munari \& Zwitter 1997) and 30 QSOs (Poznanski et al. 2012), are plotted as asterisks. The dashed line corresponds to the relation derived by Poznanski et al. (2012) based on 117 moderate-to-high-resolution observations of QSOs and nearly a million low-resolution Sloan Digital Sky Survey spectra of galaxies and QSOs. This line provides a reasonably adequate representation of the Milky Way EW(Na I D) measurements, but the rms dispersion of $0.08 \mathrm{dex}$ in $\log A_{V}$ quoted by these authors and shown in gray in Figure 9 is clearly underestimated. Presumably this is because the points used by Poznanski et al. to derive the final relations and dispersion (see Figure 9 of their paper) were obtained by averaging individual measurements of very large numbers of objects. Also plotted in Figure 9 is the curve given by Munari \& Zwitter (1997) for single-absorption components of the D1 line. As noted by Poznanski et al. (2012), this relation provides an equally good fit when scaled to match the observations. The dispersion in $A_{V}$ with respect to this latter relation is 0.30 dex, corresponding to a $68 \%$ error in $A_{V}$ for any single object rather than the $\sim 20 \%$ error implied by Poznanski et al. (2012). It is also clear from Figure 9 that the equivalent width of the $\mathrm{Na}$ I $\mathrm{D}$ lines becomes insensitive to estimating the dust extinction for EW(Na I D) $\gtrsim 1 \AA$ (see also Munari \& Zwitter 1997).

Turning to the host absorption EW(Na I D) measurements, Figure 9 reveals an effect similar to that seen in the column densities plotted in Figure 3, with a significant number of the points lying more than $3 \sigma$ above the Milky Way relationship and the majority of these belonging to the "Blueshifted" class. As more SNe Ia with good photometric and spectroscopic coverage have been observed, it has become clear that the EW(Na I D) is not a reliable indicator of the host dust extinction of SNe Ia (Blondin et al. 2009; Folatelli et al. 2010; 


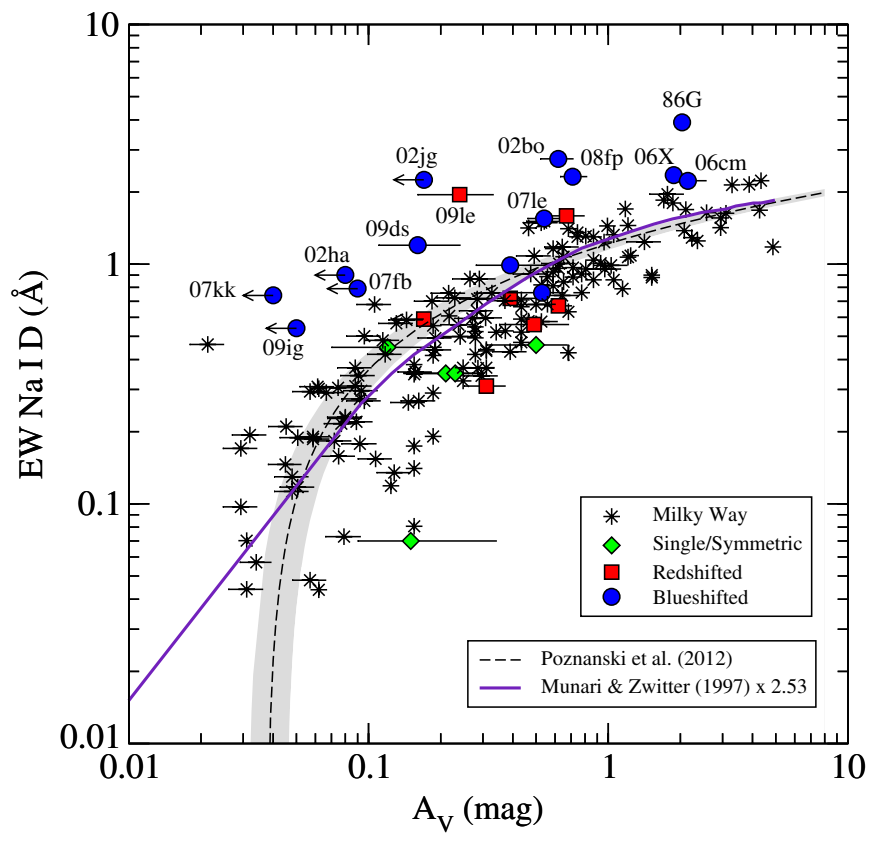

Figure 9. Na I D equivalent widths of both the Milky Way and host absorption samples are plotted vs. the dust extinction, $A_{V}$. The Milky Way sample has been augmented by high dispersion measurements of 82 stars (Sembach et al. 1993; Munari \& Zwitter 1997) and 30 QSOs (Poznanski et al. 2012). The dashed line corresponds to the Galactic relation derived by Poznanski et al. (2012), with the $1 \sigma$ dispersion found by these authors indicated by the gray shading. The solid purple line shows the relation for the D1 line found by Munari \& Zwitter (1997), multiplied by a factor of 2.53 to match the total equivalent widths plotted here. (A color version of this figure is available in the online journal.)

Poznanski et al. 2011). Figure 3 shows that the reason for this poor correlation is the relatively large fraction of $\mathrm{SNe}$ Ia with strong $\mathrm{Na}$ I $\mathrm{D}$ absorption that is an order of magnitude greater in column density than expected from the SN Ia colors and the Milky Way relation. Indeed, based on our high-dispersion observations, it would seem that the only reliable deduction that can be made using either column densities or equivalent widths is that the absence of detectable $\mathrm{Na}$ I absorption in a high signalto-noise ratio spectrum is consistent with low dust extinction.

Note that in some cases equivalent widths and column densities give quite different answers. An example is the case of SN 1986 G and SN $2006 \mathrm{~cm}$. While both of these events suffered a similar amount of dust extinction, the host absorption $\mathrm{Na}$ I column density for SN 1986G was fully consistent with the Milky Way $\log N_{\mathrm{Na}}$ versus $A_{V}$ relation, whereas for SN $2006 \mathrm{~cm}$ it was greater by $\sim 1.2$ dex (see Figure 3 ). Nevertheless, Figure 9 shows that the EW(Na I D) for SN $2006 \mathrm{~cm}$ was significantly smaller than that of SN 1986G. The explanation for this apparent inconsistency is found in Figure 10, where the Na I column density is plotted versus the equivalent width of the D lines for both the Milky Way and host absorption samples. Curves of growth for three different values of the Doppler parameter, $b$, are also displayed in this figure. These are shown for both single and multiple absorption components with the same Doppler parameter, with the single-component cases corresponding to the left-most curve for each value of $b$. Note that the curve for a single component with $b=8 \mathrm{~km} \mathrm{~s}^{-1}$ is nearly identical to the curves for two components with $b=4 \mathrm{~km} \mathrm{~s}^{-1}$ or four components with $b=2 \mathrm{~km} \mathrm{~s}^{-1}$. This ambiguity is inherent to equivalent widths, and explains why column densities are to be preferred whenever possible.

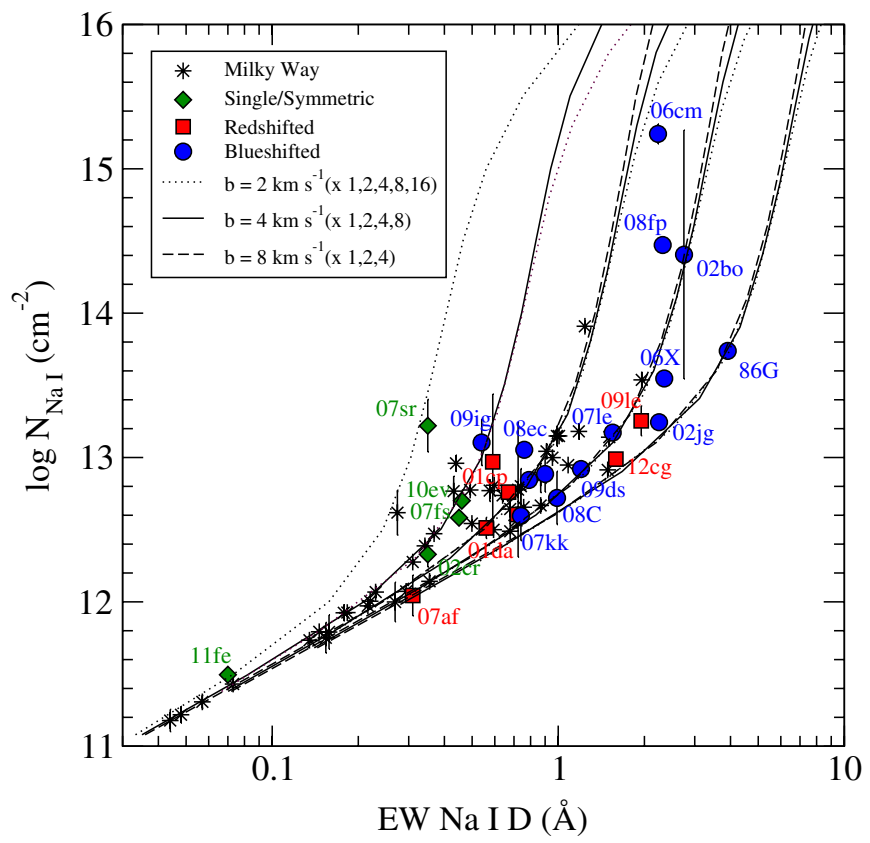

Figure 10. Na I column densities are plotted vs. Na I D equivalent widths for both the Milky Way and host absorption samples. Theoretical curve of growth relations for three different values of the Doppler parameter, $b$, are shown for reference. For each Doppler parameter, we show predictions for a single absorption component as well as for up to 16 components with the same value of $b$ (as indicated in the legend.) In all cases, the single component predictions are the left-most curves with increasing number of components lying progressively to the right.

(A color version of this figure is available in the online journal.)

Returning to SN 1986G, the profile of the host absorption D lines consisted of five major components covering a wide swath ( $\sim 200 \mathrm{~km} \mathrm{~s}^{-1}$ ) of the spectrum (D'Odorico et al. 1989). The two strongest components had Doppler parameters of $10 \mathrm{~km} \mathrm{~s}^{-1}$ and column densities $\log N_{\mathrm{Na}}$ of 13.4 and 13.1 , respectively. The third-strongest component also had $\log N_{\mathrm{Na}}=13.1$, but with $b=7 \mathrm{~km} \mathrm{~s}^{-1}$ and the two weakest both had $b \sim 5 \mathrm{~km} \mathrm{~s}^{-1}$, with column densities of 12.6 and 11.7. Reading off the equivalent widths corresponding to these individual components from Figure 10 and summing them yields a total equivalent width EW(Na I D) very close to the observed value of $3.8 \AA$. In the case of SN $2006 \mathrm{~cm}$, the Na I D profile was dominated by three major components, but concentrated into a considerably narrower $\left(\sim 60 \mathrm{~km} \mathrm{~s}^{-1}\right)$ portion of the spectrum. The two strongest components had $b=5 \mathrm{~km} \mathrm{~s}^{-1}$ and $\log N_{\mathrm{Na}}$ of 15.0 and 14.5 , respectively, while the third component was slightly narrower ( $b \sim 4 \mathrm{~km} \mathrm{~s}^{-1}$ ) with a column density of 13.7 . Summing the corresponding equivalent widths using Figure 10 gives a total equivalent width close to, but slightly larger than, the observed value, with the discrepancy accounted for by the significant overlapping in velocity space of the absorption components.

\subsection{Relation to Previous Studies of Na I D in SNe Ia}

The fact that nearly all of the SNe Ia showing anomalously large $\mathrm{NaI}$ column densities belong to the Sternberg et al. (2011) "Blueshifted" class suggests an association with CSM. Sternberg et al. (2011) estimated that a quarter to a third of local SNe Ia with detectable Na I D absorption show evidence of outflowing CSM, which is remarkably similar to the percentage of objects with anomalously large $\mathrm{Na}$ I column densities that we find in this study. It should be kept in mind, however, that the 

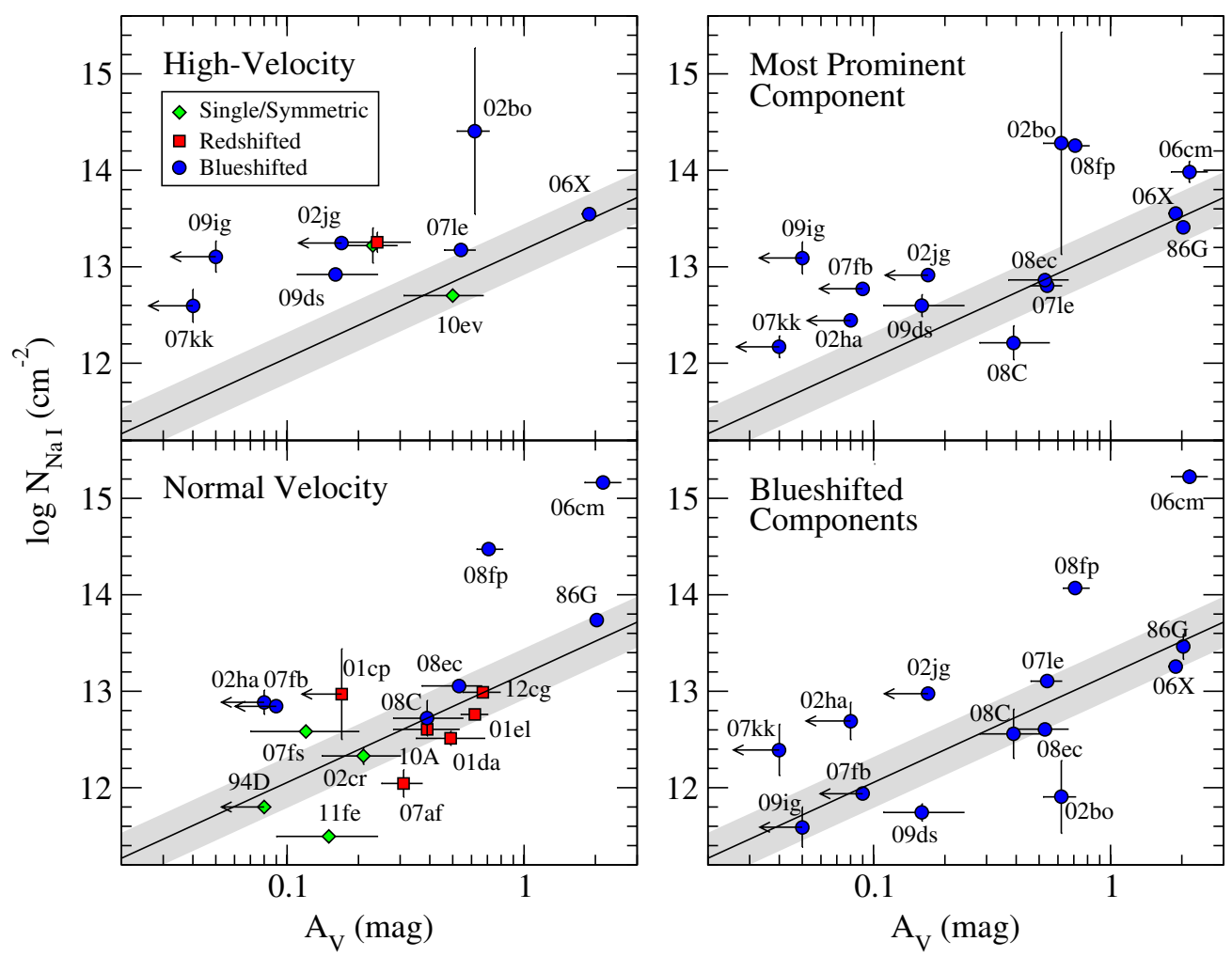

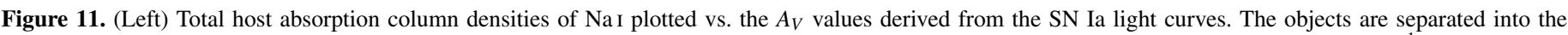

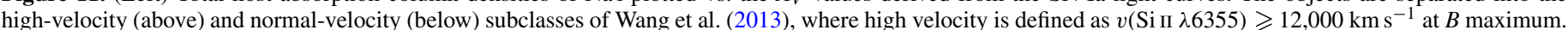

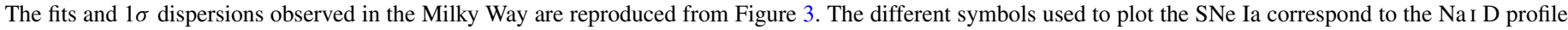

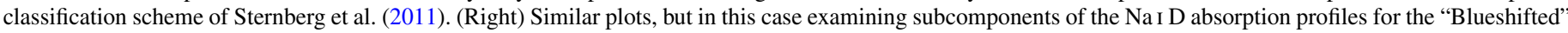

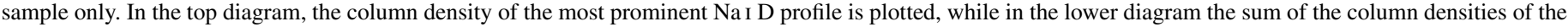
components blueward of the most prominent component are shown.

(A color version of this figure is available in the online journal.)

Sternberg et al. (2011) classifications are valid in a statistical sense only. When considering single objects, it is impossible to ascertain the systemic velocity of the progenitor and thus ascribe any particular absorption component to either inflow or outflow. Rather, it is the unexplained surplus of SNe with blueshifted absorption features that implies an association with some kind of outflow. Note that the strong preference observed for blueshifted features compared to non-blueshifted ones does not depend on whether the velocity zero point is taken to be that of the strongest absorption component or if, alternatively, it is defined with respect to emission or absorption lines in the SN spectrum due to the host galaxy or using the radial velocity of the host (Sternberg et al. 2011; Maguire et al. 2013).

Foley et al. (2012b) found that SNe Ia of the "Blueshifted" class have systematically higher ejecta velocities at maximum brightness relative to the rest of the SN Ia population, suggesting a link between the SN progenitor system and the explosion properties. Wang et al. (2013) recently presented evidence that high-velocity SNe Ia, defined as having $v(\operatorname{Si}$ II $\lambda 6355) \geqslant$ $12,000 \mathrm{~km} \mathrm{~s}^{-1}$ at $B$ maximum, are more concentrated in the inner and brighter regions of their host galaxies, and that they tend to occur in more-luminous hosts than do normal-velocity events. They suggest that high-velocity SNe represent a subtype of the Type Ia class originating from younger, more metal-rich progenitor systems. The left half of Figure 11 shows plots of $\log N_{\text {Na I }}$ versus $A_{V}$ for the SNe Ia in our host absorption sample split into the high-velocity and normal-velocity subsamples. Taken as a group, the high-velocity events deviate significantly from the Milky Way relation, and $70 \%$ of them belong to the "Blueshifted" class. The median of the absolute deviations of the high-velocity SNe Ia with respect to the Milky Way relation is 0.88 dex in column density, whereas it is 0.29 dex for the normal-velocity subsample. Nevertheless, the correlation is imperfect, with four of the most deviant "Blueshifted" SNe (2002ha, 2006 cm, 2007fb, and 2008fp) belonging to the normalvelocity subsample.

Our observations are also relevant to a recent paper by Förster et al. (2012) who found an apparent correlation between the late-phase nebular velocity shift, $v_{\text {neb }}$, and the equivalent width of the narrow host Na I D absorption as measured in lowdispersion spectra. The sense of this dependence was that $\mathrm{SNe}$ with $v_{\text {neb }}>0$ have generally stronger Na I D lines than those with $v_{\text {neb }}<0$. Maeda et al. $(2010,2011)$ had previously found a correlation of both ejection velocity and peak $B-V$ color with $v_{\text {neb }}$, which they interpreted as a viewing angle effect of offcenter explosions. Förster et al. (2012) argued that the color effect might alternatively be due to dust mixed with previouslyexisting CSM, thus accounting for not only the stronger Na I $\mathrm{D}$ lines in $\mathrm{SNe}$ with $v_{\text {neb }}>0$, but also the redder colors of these objects. Nevertheless, our discovery that $\mathrm{SNe}$ with "Blueshifted" Na I D profiles often show anomalously large Na I column densities taken together with the Foley et al. (2012b) finding that "Blueshifted" SNe Ia have systematically higher ejecta velocities and redder colors at maximum provides an alternative explanation to the $\mathrm{EW}\left(\mathrm{Na}\right.$ I D) versus $v_{\text {neb }}$ correlation that does not require a dusty CSM.

Recently, Maguire et al. (2013) presented single-epoch, intermediate-resolution $(\lambda / \Delta \lambda \sim 18,200)$ spectral observations 
Table 4

Goodness of Fit, $\chi_{v}^{2}$, to the Galactic $\log N_{\text {Na I }}$ versus $A_{V}$ Relation

\begin{tabular}{llcc}
\hline \hline & \multicolumn{1}{c}{ Sample } & All & $\begin{array}{c}\text { Excluding } \\
\text { Upper Limits }\end{array}$ \\
\hline Total Column Densities & Sembach et al. (1993) + Milky Way (this paper) & 135 & $\ldots$ \\
& LMC/SMC Cox et al. (2006); Welty et al. (2006) & 201 & $\ldots$ \\
& Single/Symmetric + Redshifted & $\geqslant 114$ & 130 \\
& Blueshifted & $\geqslant 434$ & 301 \\
Most Prominent Component & Blueshifted & $\geqslant 233$ & 60 \\
Blueshifted Components & Blueshifted & $\geqslant 179$ & 233 \\
\hline
\end{tabular}

Notes. The value of the $\chi^{2}$ per degree of freedom, $\chi_{v}^{2}$, is calculated with respect to the fit to the combined sample of the Sembach et al. (1993) data and the Milky Way measurements given in this paper (Equation (4)).

for a sample of $17 \mathrm{SNe}$ Ia, which they then combined with the sample of high-resolution spectra published by Sternberg et al. (2011). These authors confirmed the Sternberg et al. (2011) finding that an excess of events showed blueshifted structure in the $\mathrm{Na}$ I $\mathrm{D}$ lines, and also pointed out that the strength of the $\mathrm{Na}$ I D absorption was stronger in SNe Ia displaying blueshifted profiles than in those without such structure, in accordance with our own findings. In addition, Maguire et al. (2013) found that the strength of the blueshifted subcomponent of the D lines for those objects with blueshifted profiles was correlated with the $(B-V)$ colors of the $\mathrm{SNe}$ at maximum light. These findings were interpreted as being strongly suggestive of absorbing material (CSM) near the SN. Maguire et al. (2013) went on to speculate that $\mathrm{SNe}$ Ia with strong, blueshifted absorption may represent a distinct population of events produced preferentially in late-type galaxies.

In the upper right half of Figure 11, the column densities of the most prominent (deepest) component of the host Na I D absorption for each "Blueshifted" $\mathrm{SN}$ in our sample are plotted versus the values of $A_{V}$ inferred from the SN colors. The column densities were derived from the VPFIT results shown in Figures 1 and 2, and correspond to the zero velocity components. Following Maguire et al. (2013), we also plot in the lower right half of Figure 11 the sum of the column densities of the absorption components lying blueward of the most prominent component, again for the "Blueshifted" SNe in our sample. ${ }^{29}$ In both cases, a general correlation is observed, but with considerable scatter. To quantify the goodness of fit to the Galactic $N_{\mathrm{NaI}}$ versus $A_{V}$ relationship (Equation (4)), we calculate the chi-squared per degree of freedom, $\chi_{v}^{2}$, for several different samples in Table 4 . In the case of the SNe, the calculation is done both including the objects with upper limits for $A_{V}$, and excluding them. Note that all of the $\chi_{v}^{2}$ values are large - even for the Sembach et al. (1993) + Milky Way sample that was used to derive Equation (4) - reflecting the large intrinsic dispersion in the $N_{\mathrm{Na}}$ versus $A_{V}$ relation, which is considerably greater than the individual errors in the column densities. Interestingly, the value for the combined sample of "Redshifted" and "Single/Symmetric" SNe is similar to that of the Sembach et al. (1993) + Milky Way sample, implying that

\footnotetext{
29 Note that rather than using the most prominent absorption component to define zero velocity, Maguire et al. (2013) employed galaxy emission or absorption lines observed in the SN Ia spectrum or, lacking this, the recessional velocity of the host galaxy. While both approaches have their merits, they do not result in significant differences in defining the blueshifted absorption components.
}

their column densities and dust extinctions are fully consistent with the Galactic relation. On the other hand, $\chi_{v}^{2}$ is very large for "Blueshifted" $\mathrm{SNe}$, consistent with the significant number of objects with anomalously large values of $N_{\mathrm{NaI}}$. Table 4 shows that using either the column density of the most prominent (zero velocity) absorption component or the sum of the column densities of the absorption components blueward of zero velocity for the "Blueshifted" $\mathrm{SNe}$ gives $\chi_{v}^{2}$ values that, while lower than that obtained for the total column densities, are still relatively large. Our conclusion is that splitting the "Blueshifted" SNe into these two subsamples of the absorption components does not provide any particular insight into the location of the absorbing gas and dust.

If the strong $\mathrm{Na}$ I absorption is an indication of outflowing CSM, it is somewhat puzzling that the three "Blueshifted" $\mathrm{SNe}$ Ia in our sample that almost certainly had CSM- SNe 2006X, 2007le, and 2012cg-did not show unusually large $\mathrm{Na}$ I column densities for the host extinction implied by their colors (see Figure 3). Echelle spectroscopy of these three $\mathrm{SNe}$ revealed variations in the strengths of certain components of the Na I D lines (Patat et al. 2007; Simon et al. 2009; S. Raskutti et al. 2013, in preparation). The evidence strongly suggests that the variations were due to changing ionization conditions in CSM, particularly since similar variations were not observed for the Ca II $\mathrm{H} \& \mathrm{~K}$ lines. Multiple echelle spectra were not obtained for the majority of the $\mathrm{SNe}$ Ia in our sample, and so we do not know if more objects might have shown variable Na I absorption. An exception is SN 2008fp, one of the SNe with the strongest Na I lines. Cox \& Patat (2013) did not find evidence for variability of any of the absorption components in this SN from echelle spectra obtained at $+6,+11,+17$, and +39 days after maximum light. Of course, whether or not variability is observed in any particular object will be a function of the density, distribution, and geometry of the CSM, and also the timing of the observations. Perhaps in the case of SN 2008fp, the variations were too small to detect or occurred before the first spectrum was obtained. High signal-to-noise ratio observations beginning at the earliest epochs will be necessary to make more progress on this question.

The rare class of 2002ic-like events that display a strong CSM interaction, for which a white dwarf with a massive asymptotic giant branch (AGB) star companion (Hamuy et al. 2003) or a symbiotic nova (Dilday et al. 2012) have been proposed as progenitor systems, may be key objects for unraveling the CSM-progenitor connection. Echelle spectroscopy of the best-observed member of this class, PTF11kx (Dilday et al. 
2012), obtained on four epochs ranging from -1 to +44 days with respect to optical maximum revealed strong variations in the strength of the most blueshifted component of the Na I D lines. Our analysis of these spectra gives a total column density of $\log N_{\mathrm{Na}}=12.93 \pm 0.19 \mathrm{~cm}^{-2}$ and a $3 \sigma$ upper limit of $\operatorname{EW}(5780)<40 \mathrm{~m} \AA$. Dilday et al. (2012) estimated a dust extinction of $A_{V} \sim 0.5 \mathrm{mag}$ from the SN colors before the strong CSM interaction set in. These numbers place PTF11kx squarely on the Galactic $N_{\mathrm{Na}}$ versus $A_{V}$ trend, and only slightly below the $\mathrm{EW}(5780)$ versus $A_{V}$ relation.

\subsection{SNe 2006cm, 2008fp, 2009ig, and 2009le}

Three of the best-observed examples of "Blueshifted" SNe Ia in our sample with anomalously large $\mathrm{Na}$ I column densities are $2006 \mathrm{~cm}, 2008 \mathrm{fp}$, and $2009 \mathrm{ig}$. In this section, we examine some of their properties in more detail along with those of the "Redshifted" SN 2009le.

SNe $2006 \mathrm{~cm}$ and 2008fp were quite similar in their spectral and photometric characteristics. Both were substantially reddened by dust $-E(B-V)_{\text {host }}=1.08$ and 0.58 , respectivelyand both showed strong, saturated Na I D host absorption. Figure 4 shows the Na I D1 and K I $\lambda 7665$ line profiles of these two SNe. Cox \& Patat (2013) recently presented an in-depth study of the host interstellar absorption features in SN 2008fp. They found a rich spectrum of narrow atomic and molecular lines characteristic of a cold, translucent cloud, and argued that the host dust extinction for this $\mathrm{SN}$ is produced in this cloud. This system is responsible for the $\mathrm{K}_{\mathrm{I}}$ absorption at zero velocity in Figure 4 as well as the blue half of the strongly saturated component of the Na I D lines observed between -20 and $+40 \mathrm{~km} \mathrm{~s}^{-1}$. In modeling the latter absorption "trough" with VPFIT, we have used not only the information provided by the $\mathrm{K}$ I lines, but also the results of Cox \& Patat (2013) — in particular, the velocity and column density of the Na I UV $\lambda \lambda 3302,3303$ doublet and the velocities of the two components of the Fe I absorption observed by these authors. The fits yield $\log N_{\mathrm{Na} \text { I }}=14.43 \pm 0.04 \mathrm{~cm}^{-2}$ and $\log \left(N_{\mathrm{Na}_{\mathrm{I}}} / N_{\mathrm{K}}\right) \sim 2.4$ for the "trough." Measurement with VPFIT of the host $\mathrm{Ca}$ II $\mathrm{H} \& \mathrm{~K}$ absorption over this same velocity range yields $N_{\mathrm{Na}} / N_{\mathrm{Ca} \text { II }} \sim 13$, typical of cold gas in the disk of the Milky Way (Siluk \& Silk 1974; Vallerga et al. 1993).

The evidence thus points to the ISM of the host galaxy of SN $2008 \mathrm{fp}$ as having produced at least a significant fraction of the strongly saturated $\mathrm{Na}$ I D lines. The origin of the blueshifted host absorption component at $-44 \mathrm{~km} \mathrm{~s}^{-1}$ is less clear. We measure $\log N_{\mathrm{Na}}=13.43 \pm 0.14 \mathrm{~cm}^{-2}, \log \left(N_{\mathrm{Na}} / N_{\mathrm{KI}}\right) \sim 2.5$, and $N_{\mathrm{NaI}} / N_{\mathrm{Ca} \text { II }} \sim 110$. The latter value suggests that this absorption is not produced in a high velocity cloud since, in the Milky Way, these typically have $N_{\mathrm{Na}} / N_{\text {Ca II }} \lesssim 1$ (Siluk \& Silk 1974; Vallerga et al. 1993).

Cox \& Patat (2013) also studied the host DIB lines in the spectrum of SN 2008fp, which they found to be slightly redshifted by $\sim 20 \mathrm{~km} \mathrm{~s}^{-1}$ with respect to the strong atomic and molecular lines. This is consistent with our own measurement of the velocity of the host $5780 \AA$ DIB in SN 2008fp which is very similar to the velocity of the most redshifted component that we infer for the Na I D lines (see Figure 4). However, the $5780 \AA$ feature is broad (see Figure 6) and radial velocity measurements are likely to be sensitive to the exact profile used to determine the observed wavelength, which is not a simple Gaussian as we assume in this paper (Galazutdinov et al. 2008). The rest wavelength of the $5780 \AA$ DIB is also not very accurately known, with the uncertainty corresponding to $10-15 \mathrm{~km} \mathrm{~s}^{-1}$ in radial velocity (e.g., Galazutdinov et al. 2000; Tuairisg et al. 2000;
Hobbs et al. 2008, 2009). Finally, the measured velocity of the DIB absorption will depend on the contributions of the various clouds in the line-of-sight, but this is impossible to decipher due to the significant width of the DIBs. Hence, the fact that the velocity of the $5780 \AA$ DIB in SN 2008fp does not coincide in redshift with the cloud responsible for the strongest component of the $\mathrm{K}_{\mathrm{I}}$ lines should probably not be given too much weight.

In the case of $\mathrm{SN} 2006 \mathrm{~cm}$, three absorption systems are observed for the $\mathrm{K}_{\mathrm{I}} \lambda 7665$ line (see Figure 4). Using the velocities and $b$ values of these as a guide to fitting the $\mathrm{Na}$ I D lines, we find $\log N_{\mathrm{Na}}=15.23 \pm 0.07 \mathrm{~cm}^{-2}$ and $\log \left(N_{\mathrm{Na}} / N_{\mathrm{K}_{\mathrm{I}}}\right) \sim 2.9$ for the strong absorption between -50 and $+10 \mathrm{~km} \mathrm{~s}^{-1}$. As shown in Figure 4 , the velocity measured for the host $5780 \AA$ A DIB falls near the center of the D line absorption. SN $2006 \mathrm{~cm}$ was located only 2 arcsec from the center of its host, the edge-on Sb galaxy UGC 11723, and our spectrum contains weak, tilted emission lines of $\mathrm{H} \alpha$ and [N II] $\lambda \lambda 6548,6584$ along the slit, presumably due to diffuse ionized gas in the host. The FWHM velocity of this emission in the extracted spectrum of the $\mathrm{SN}$ is indicated in Figure 4, and closely coincides with the "trough" of Na I absorption.

SN 2009ig is especially interesting since its colors are consistent with little or no host reddening, yet its spectrum displays strong $\mathrm{Na}$ I D host absorption (see Figure 7). Three other SNe in our sample-2002ha, 2007fb, and 2007kk-share this characteristic, and low-dispersion spectra have identified similar cases-e.g., SNe 2006dd and 2006mr in NGC 1316 (Stritzinger et al. 2010). The observed column density of Na I for SN 2009ig implies a dust extinction in the range $A_{V}=$ 0.5-1.2 mag using the Milky Way relation given in Section 3, yet both the SN colors and the upper limit on the strength of the host absorption DIB at $5780 \AA$ argue for $A_{V}<0.1$ mag. Host $\mathrm{Ca}$ II $\mathrm{H} \& \mathrm{~K}$ absorption is also present in our spectrum at a ratio $N_{\mathrm{NaI}} / N_{\mathrm{Ca} \text { II }} \sim 3$, typical of cold gas in the disk of the Milky Way (Siluk \& Silk 1974). Whatever the source (CSM or ISM) of the gas producing the strong $\mathrm{Na}$ I and $\mathrm{Ca}$ II absorption in this $\mathrm{SN}$, the dust-to-gas ratio must be exceptionally low.

SN 2009le is a "Redshifted" SN Ia with a total host Na I column density more than $2 \sigma$ above the Galactic $N_{\text {Na I versus }} A_{V}$ relation. The lower-right plot in Figure 5 shows that it is similar to SN 2009ig in having a large host Na I column density, but relatively weak $5780 \AA$ DIB feature. At $\log \left(N_{\mathrm{Na}_{\mathrm{I}}} / N_{\mathrm{K}}\right) \sim 2.1$, the $N_{\mathrm{Na}} / N_{\mathrm{K}}$ ratio is somewhat high, although not extraordinarily so (see Section 4.5). Figure 12 shows the observed Na I D1 and K I $\lambda 7665$ profiles of SN 2009le, along with the VPFIT model. The host Na I absorption is quite unusual in covering $\sim 250 \mathrm{~km} \mathrm{~s}^{-1}$ in velocity space, more than any other $\mathrm{SN}$ in our sample. Weak emission lines of $\mathrm{H} \alpha, \mathrm{H} \beta,[\mathrm{N}$ II] $\lambda \lambda 6548,6584$, and [S II] $\lambda \lambda 6717,6731$ are observed along the slit in the spectrum of the $\mathrm{SN}$. The $[\mathrm{N}$ II $] \lambda 6584 / \mathrm{H} \alpha$ and $[\mathrm{S}$ II $] / \mathrm{H} \alpha$ flux ratios are both $\sim 0.7$ and the $[\mathrm{O}$ III $] \lambda 5007$ line is not detected, suggesting that the ionized gas is reasonably metal-rich (Pettini \& Pagel 2004). The FWHM velocity range of the $\mathrm{H} \alpha$ and [N II] emission in the extracted spectrum of the $\mathrm{SN}$ is indicated in Figure 12, as is the heliocentric systemic velocity of $5,334 \mathrm{~km} \mathrm{~s}^{-1}$ of the host galaxy ESO 478-G006 (Springob et al. 2005).

The Na I absorption extending from +80 to $+200 \mathrm{~km} \mathrm{~s}^{-1}$ in SN 2009le may be due to high velocity clouds in the host galaxy since $N_{\mathrm{NaI}} / N_{\mathrm{Ca} \text { II }}<2$ for most of these components (Siluk \& Silk 1974; Vallerga et al. 1993), whereas the stronger absorption between -50 and $+80 \mathrm{~km} \mathrm{~s}^{-1}$ has $N_{\mathrm{NaI}} / N_{\mathrm{Ca} \text { II }} \sim 5$, typical of cool dense gas in the disk of the Milky Way. We find $\log \left(N_{\mathrm{Na}} / N_{\mathrm{KI}}\right)=1.7$ for the zero velocity component, 


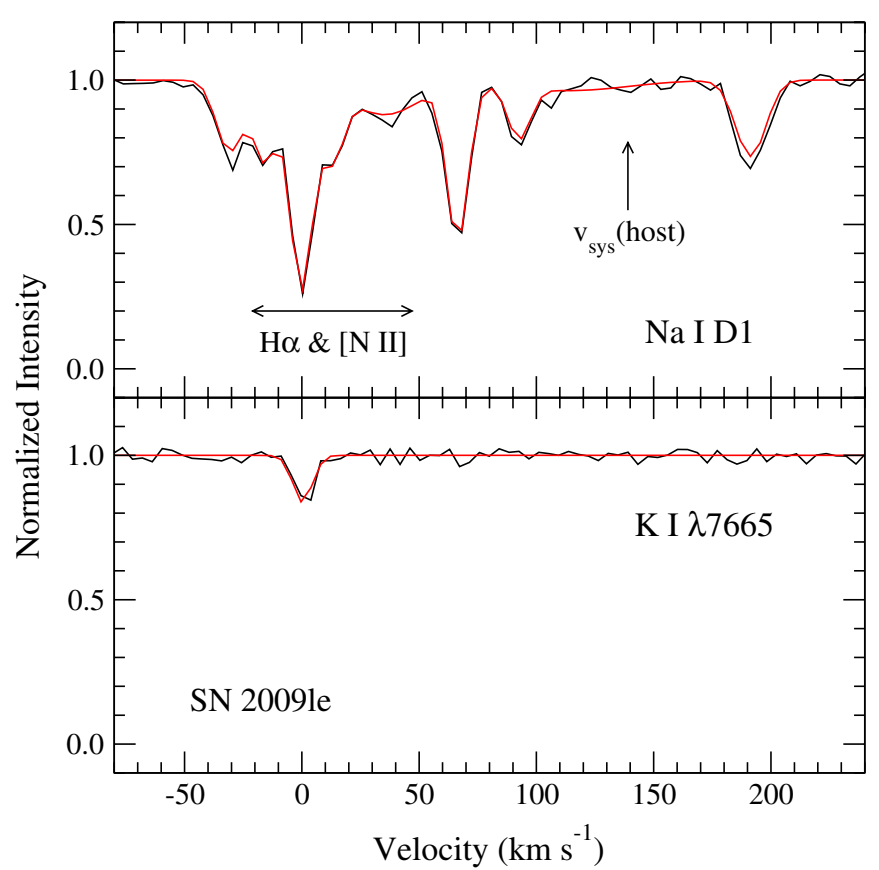

Figure 12. Host $\mathrm{NaI} D 1$ and $\mathrm{K}_{\mathrm{I}} \lambda 7665$ absorption in SN 2009le. The observations correspond to the black line and the best-fitting profile VPFIT model is shown in red. Zero velocity has been arbitrarily set to correspond to the single component observed for of the K I $\lambda 7665$ line. The FWHM velocity range covered by $\mathrm{H} \alpha$ and $[\mathrm{N} \mathrm{II}]$ emission in the extracted spectrum of the $\mathrm{SN}$ is indicated. The systematic velocity of the host galaxy is also shown.

(A color version of this figure is available in the online journal.)

consistent with the Milky Way ratio. If we remove the highvelocity components from consideration, the host $\mathrm{Na}$ I column density decreases by only $\sim 0.1 \mathrm{dex}$, and so the strength of the D-line absorption in this SN remains strong, although not exceptionally so, for the amount of dust extinction implied by both the colors and the equivalent width of the $5780 \AA$ DIB.

$$
\text { 4.5. The } N_{\mathrm{Na}} / N_{\mathrm{KI}} \text { Ratio }
$$

In the Milky Way ISM, the Na I and K I column densities are tightly correlated as would be expected based on ionization potentials and condensation temperatures (Welty \& Hobbs 2001). This is illustrated in the left half of Figure 13 which shows the measurements of Welty \& Hobbs (2001) and Kemp et al. (2002). A fit to these data gives $\log \left(N_{\mathrm{Na}} / N_{\mathrm{K}}\right)=1.9$, with an rms dispersion of 0.3 dex. Kemp et al. (2002) argued from their observations that the value of the $N_{\mathrm{Na}} / N_{\mathrm{K}}$ ratio actually increases slightly with column density as indicated by the thick black line in Figure 13. This is a small effect that is also clearly consistent with the data. Included in Figure 13 are $\mathrm{Na} I$ and $\mathrm{KI}$ column density measurements in the LMC (Cox et al. 2006; Welty et al. 2006) and SMC (Welty et al. 2006), which give a slightly lower value of $\log \left(N_{\mathrm{Na}} / N_{\mathrm{K}}\right)=1.6$.

Our observations of the Milky Way sample in Table 1 are plotted in the right half of Figure 13, and give a weighted mean of $\log \left(N_{\mathrm{Na}} / N_{\mathrm{KI}}\right)=1.4$ with an rms of 0.3 . This lower value is likely due to saturation effects in our measurements of the D lines (see Section 2.1) since the Galactic measurements of Welty \& Hobbs (2001) and Kemp et al. (2002) were derived from the UV $\lambda \lambda 3302,3303$ doublet for $\log N_{\mathrm{Na}}>12.5 \mathrm{~cm}^{-2}$. The weighted mean for the SN Ia host absorption sample is $\log \left(N_{\mathrm{Na}} / N_{\mathrm{K}_{\mathrm{I}}}\right)=1.7$ with an rms of 0.6. Excluding the three $\mathrm{SNe}$ with anomalously large Na I column densities-2006 cm, 2008fp, and 2009ig-lowers this to 1.6 with an rms of 0.3 .
The $\log \left(N_{\mathrm{Na}} / N_{\mathrm{KI}}\right)$ values for SNe $2006 \mathrm{~cm}$ and $2008 \mathrm{fp}$ of 2.9 and 2.4 , respectively, are significantly greater than the mean our spectra give for the Milky Way. Interestingly, however, the measurements for SNe 2009ig and 2009le are fully consistent with the Galactic $N_{\mathrm{NaI}} / N_{\mathrm{K}}$ ratio.

Kemp et al. (2002) argue on theoretical grounds that, for a cosmic abundance ratio of $N_{\mathrm{Na}} / N_{\mathrm{K}} \approx 15$, standard values of the photoionization rate and recombination coefficients, and electron densities between 0.001 and $1 \mathrm{~cm}^{-3}$, the $N_{\mathrm{Na}} / N_{\mathrm{K}}$ ratio should be in the range of $\approx 60-80$. Although at low column densities a harder radiation field could lower the ratio close to the cosmic abundance value of 15 , at the highest column densities (i.e., as the electron density approaches zero), the $N_{\mathrm{Na}} / N_{\mathrm{K}}$ ratio should asymptotically approach a value of $\approx 80$, or $\log \left(N_{\mathrm{Na}} / N_{\mathrm{KI}}\right) \approx 1.9$. Observations in the Milky Way show exactly this effect (see Figure 5 of Kemp et al. (2002)), although a few sight-lines reach values as large as $\log \left(N_{\mathrm{Na}_{\mathrm{I}}} / N_{\mathrm{KI}}\right) \sim 2.1$.

Thus, the much higher values of $\log \left(N_{\mathrm{Na}} / N_{\mathrm{KI}}\right)=2.4-2.9$ observed for the host absorption in SNe $2006 \mathrm{~cm}$ and $2008 \mathrm{fp}$ imply either an enhancement of $\mathrm{Na}$ I or a depletion of $\mathrm{K}_{\mathrm{I}}$. The fact that both the DIBs and $\mathrm{K}_{\mathrm{I}}$ column densities for these two objects are more consistent with the value of $A_{V}$ derived from the $\mathrm{SN}$ colors than are the $\mathrm{Na}$ I column densities would seem to suggest that an enhanced abundance of $\mathrm{Na}$ I is the more likely explanation. More observations of the $\mathrm{K}_{\mathrm{I}}$ doublet in $\mathrm{SNe}$ Ia are needed to determine if the large $N_{\mathrm{Na}} / N_{\mathrm{K}}$ ratios observed for $\mathrm{SNe} 2006 \mathrm{~cm}$ and 2008fp are a common characteristic of SNe Ia with anomalously strong $\mathrm{Na}$ I $\mathrm{D}$ lines, or whether most, like $\mathrm{SNe} 2009 \mathrm{ig}$ and 2009le, show normal $N_{\mathrm{Na}} / N_{\mathrm{KI}}$ ratios.

\subsection{Possible Origins of the Strong Na I D Absorption}

Although one-fourth of the SNe Ia in our sample displayed anomalously large host $\mathrm{Na}$ I column densities in comparison with dust reddening deduced from their colors, the observations provide conflicting clues as to the origin of this phenomenon. The fact that all such SNe have "Blueshifted" D-line profiles as classified by Sternberg et al. (2011) is highly suggestive that an outflowing CSM is responsible for the strong Na I absorption. The existence of events such as SN 2009ig that were essentially unreddened, as indicated by both their colors and the weakness of the DIB $5780 \AA$ absorption, yet showed strong host $\mathrm{Na}$ I $\mathrm{D}$ lines is also difficult to understand in the ISM scenario. Nevertheless, as discussed in Section 4.4, the evidence seems to clearly favor an ISM origin for most, if not all, of the strong D-line absorption observed in SN 2008fp, and, as detailed in Section 4.3, unusually strong Na I D lines have not been observed in the few SNe Ia studied to date for which there is independent evidence for the existence of CSM.

If all $\mathrm{SNe}$ Ia have the same progenitor systems, and if the anomalously large host Na I column densities are due to CSM, then we would expect to see strong D-line absorption in at least some of the $\mathrm{SNe}$ Ia that occur in elliptical galaxies (i.e., where there is no significant ISM). Unfortunately, only one of the SNe in our sample (2007on) occurred in a host galaxy that is classified as an elliptical. Sternberg et al. (2011) obtained high-dispersion spectra of an additional two SNe Ia (2006ct and 2006eu) in elliptical galaxies. In none of these three cases was $\mathrm{Na}$ I absorption detected. It is important to continue to obtain echelle spectra of more SNe Ia in nearby ellipticals to build up a statistically-significant sample. If it is found that $\mathrm{SNe}$ Ia in elliptical hosts never show strong $\mathrm{Na}$ I D lines, that would in turn imply that either the large Na I column densities seen in some SNe Ia are not due to CSM, or the progenitors of SNe Ia 


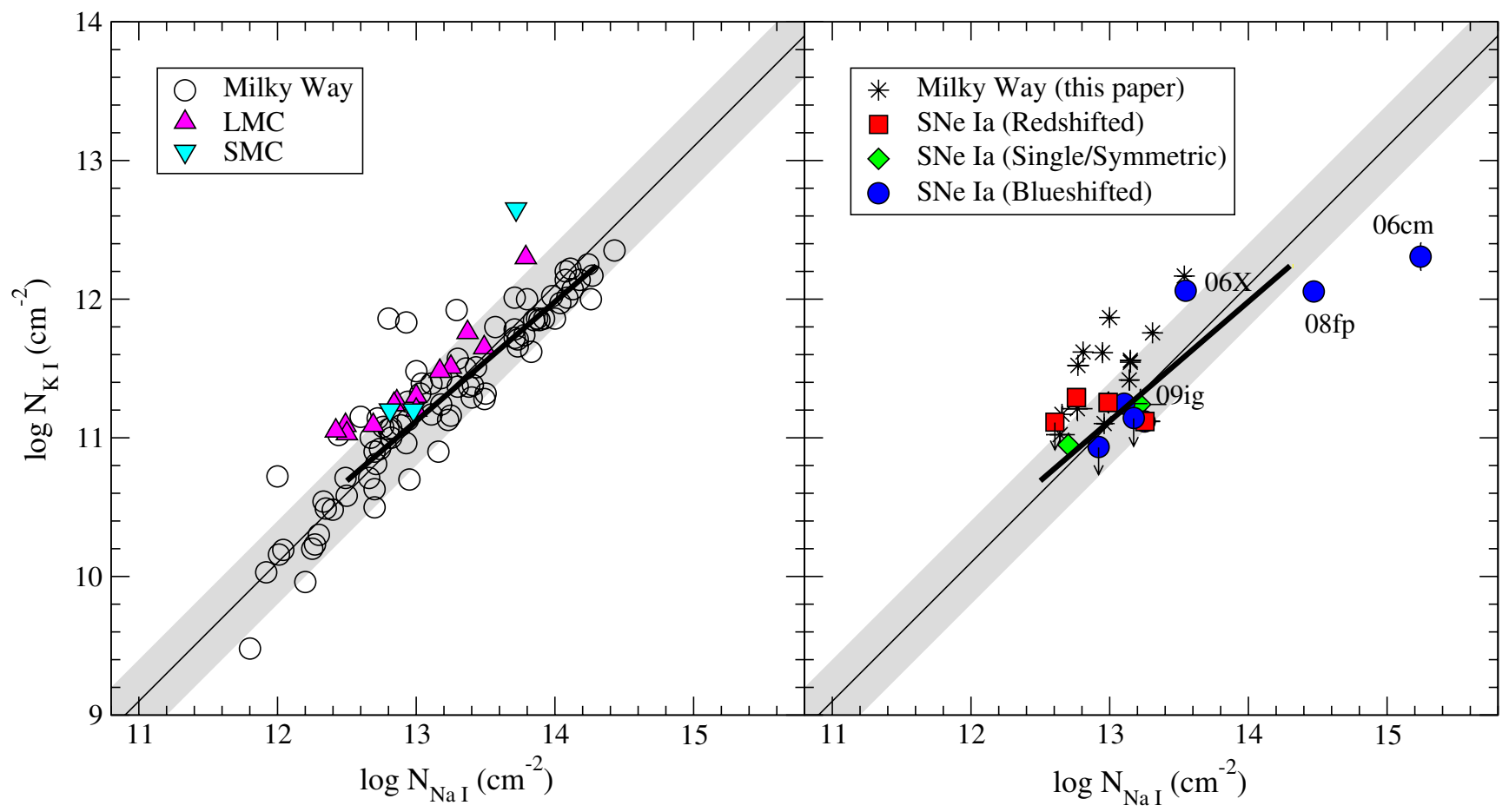

Figure 13. Column densities of Na I and K I plotted against each other. (Left) Measurements for the Milky Way (Welty \& Hobbs 2001; Kemp et al. 2002), LMC (Cox et al. 2006; Welty et al. 2006), and SMC (Welty et al. 2006) are compared. The diagonal line shows the average value of $\log \left(N_{\mathrm{Na}} / N_{\mathrm{KI}}\right)=1.9$ and rms dispersion of 0.3 dex obtained for the Milky Way measurements. The thick black line is a representation of the $\log N_{\mathrm{K}}$ vs. $\log N_{\mathrm{Na}}$ trend shown in Figure 2 of Kemp et al. (2002). (Right) Plot of column densities of Na I and K I for our Milky Way and SNe Ia host absorption samples. The fit and rms dispersion of the Milky Way stellar sample from the left half of the figure is duplicated for comparison. Also repeated is the $\log N_{\mathrm{K}_{\mathrm{I}}}$ vs. $\log N_{\mathrm{Na}}$ trend from Kemp et al. (2002).

(A color version of this figure is available in the online journal.)

in elliptical galaxies are fundamentally different from (or, are a subset of) those in later-type galaxies. Some support for the latter hypothesis is found in the observation that early-type galaxies tend to produce a factor of $\sim 20$ less SNe Ia (normalized to stellar mass) than do late-type galaxies (Mannucci et al. 2005), and that these $\mathrm{SNe}$ are, on average, faster-declining and less-luminous than those observed in spirals (Hamuy et al. 1995, 1996).

Novae have long been considered to be possible progenitors of some $\mathrm{SNe} \mathrm{Ia}$, and there is evidence, both observational and theoretical, that highly enhanced $\mathrm{Na}$ is produced in the thermonuclear runaway that powers novae outbursts. In a review of abundance determinations for novae, Gehrz et al. (1998) identified several novae whose $\mathrm{Na}$ abundances were derived from emission-line analysis to be more than an order of magnitude enhanced over solar values. It is therefore possible in the SD scenario that very high $\mathrm{Na}$ abundances in SNe Ia might be understood in terms of their having been produced in prior nova outbursts in progenitors whose eventual collapse formed the supernovae.

Giant stars in globular clusters show large $\mathrm{Na}$ abundance enhancements that are not observed in field giants (e.g., see Sneden et al. 2004, and references therein). The $\mathrm{Na}$ and $\mathrm{O}$ abundances are anti-correlated, consistent with proton-capture fusion processes during CNO burning. These abundance variations most likely result from pollution by an earlier generation of more massive stars. Similar star-to-star abundance variations in C, $\mathrm{N}, \mathrm{O}$ and $\mathrm{Na}$ have been observed in stars at or near the mainsequence turn-off (Gratton et al. 2001; Ramírez \& Cohen 2003; James et al. 2004; Cohen \& Meléndez 2005; Cohen et al. 2005), in support of the self-pollution scenario as first proposed by Cottrell \& Da Costa (1981). Perhaps, then, the "Blueshifted" profiles and anomalously large Na I column densities (with re- spect to $\mathrm{K}_{\mathrm{I}}$ ) observed in $\mathrm{SNe}$ Ia such as $2006 \mathrm{~cm}$ and $2008 \mathrm{fp}$ point to a SD progenitor system with a massive AGB star as the donor star.

The stellar yields of Na from intermediate-mass AGB stars of solar metallicity show only relatively minor enhancements of 0.2-0.4 dex (Karakas 2010; Karakas et al. 2012). This is down from the results published in Karakas \& Lattanzio (2007), which predict yields of $\mathrm{Na}$ that are up to a factor of $\approx 8$ higher (for a $6 M_{\odot}, Z=0.02$ model). The cause of the variation is the use of revised experimental reaction rates for the ${ }^{23} \mathrm{Na}+p$ reactions (see discussion in Karakas 2010). Karakas et al. (2012) varied the mass-loss rate and reaction rates, key uncertainties in AGB nucleosynthesis calculations of intermediate-mass stars. They find that the only way to obtain $\mathrm{Na}$ enrichments of $\approx 1 \mathrm{dex}$ is to use the old rates for the ${ }^{23} \mathrm{Na}+p$ reactions combined with a lower mass-loss rate in stars of mass 5-7 $M_{\odot}$ at $Z=0.02$. In summary, fine-tuning of model parameters is required in order to produce substantial $\mathrm{Na}$ enrichment $(\approx 1 \mathrm{dex})$ in the wind.

There is another way to obtain substantial enrichments in $\mathrm{Na}$. Most of the Na produced during the AGB phase remains in the core of the star. Sodium is produced by $\mathrm{H}$-shell burning but it is not destroyed by $\alpha$-captures during helium burning. For this reason, a reservoir of $\mathrm{Na}$ builds up in the outer layers of the $\mathrm{H}$-exhausted core and only a small fraction of this is mixed to the surface where it can be lost by stellar winds. By the beginning of the AGB phase, the amount of $\mathrm{Na}$ is $\gtrsim 7$ times the initial value in the outer $\lesssim 0.01 M_{\odot}$ layers of the core. This is a robust prediction that does not depend on reaction rates or initial stellar mass, and is found for a range of masses from 1.9-7 $M_{\odot}$ at $Z=0.02$. By the end of the AGB phase, this increases to 8 to 30 times the initial $\mathrm{Na}$, depending on the details of AGB evolution. Higher values are in fact found in the lowest mass 
AGB star cores of $\approx 1.9-3 M_{\odot}$. This is because of the formation of a ${ }^{13} \mathrm{C}$ pocket, a necessary ingredient for the production of $s$-process elements (see e.g., Mowlavi 1999; Cristallo et al. 2009; Karakas 2010). The Na produced in this manner will remain in the white dwarf (WD) after the AGB phase ends. This means that less fine tuning is required to produce substantial $\mathrm{Na}$ enrichment in SNe Ia with DD progenitors, assuming some of the material from the outer layers of the disrupted WD can form CSM (Raskin \& Kasen 2013; Shen et al. 2013). If the WD merger involves a He WD plus a C-O WD, then stellar models also predict $\mathrm{Na}$ enhancements of a factor of seven spread over a larger $\approx 0.1 M_{\odot}$ of the helium core $^{30}$.

\subsection{Location of the Dust and the Nature of the Low Values of $R_{V}$}

Our finding that the strength of the DIB at $5780 \AA$ correlates well with the dust extinction derived from the SN Ia colors provides an important clue to the location of the dust. DIBs are weak or absent in the CSM of nearly all mass-losing stars, including post-AGB stars (Le Bertre \& Lequeux 1993; Luna et al. 2008). Most likely this means that either the molecular carriers responsible for the DIBs are not present in the CSM, or that the excitation conditions are not what is required to produce observable absorption. If the latter were true, it is conceivable that a SN Ia exploding within a dusty CSM might produce the conditions to make DIBs visible. However, with the exception of SN 1986G which may have been located behind the dust lane of NGC 5128, all of the significantly reddened $\mathrm{SNe}$ Ia in our sample exploded in the dusty disks of their host galaxies. Thus, the simplest and most obvious interpretation of the correlation of $\mathrm{EW}(5780) \AA$ with $A_{V}$ is that most of the dust extinction is interstellar in origin, and is not produced in CSM associated with the $\mathrm{SN}$ progenitor system.

The very low values of $R_{V}$ that seem to characterize the dust reddening in many $\mathrm{SNe}$ Ia are unusual in the Milky Way, but not completely without precedent. A well-studied example is the B3 V star, HD 210121 for which photometry and spectropolarimetry give $R_{V} \sim 2.0$ (Larson et al. 1996; Fitzpatrick \& Massa 2007). The dust reddening of this star is produced by a translucent molecular cloud at high Galactic latitude characterized by an enhanced relative abundance of small grains and a dust-to-gas ratio $\sim 30 \%$ smaller than the standard Galactic value (Larson et al. 2000). Similarly low values of $R_{V}$ have been observed in a few other high Galactic latitude clouds (Larson \& Whittet 2005) and toward the Galactic bulge (Udalski 2003; Nataf et al. 2013). In their study of anomalous extinction sight-lines, Mazzei \& Barbaro (2011) list two stars with $R_{V}=0.60 \pm 0.18$ (HD 1337) and $1.10 \pm 0.18$ (HD 137569). Nevertheless, such extreme values are rare in the solar neighborhood. In their study of 328 Galactic extinction curves of $\mathrm{O}$ and $\mathrm{B}$ stars at a median distance of $\sim 1.3 \mathrm{kpc}$, Fitzpatrick \& Massa (2007) found that less than 3\% had $R_{V}<2.5$.

For comparison, four of the five $\mathrm{SNe}$ Ia in our sample with $E(B-V)>0.4$ had $R_{V}<2.5$. The average value of $R_{V}$ for these four $\mathrm{SNe}$ is $\sim 1.4$. Such unusually low values led Wang (2005) and Goobar (2008) to suggest that $R_{V}$ for these reddened $\mathrm{SNe}$ Ia is modified by multiple scattering of photons in a dusty CSM which effectively steepens the extinction law. However, this idea appears to be at odds with our finding that

\footnotetext{
30 From a $1.9 M_{\odot}$ stellar model of $Z=0.02$ which produces a $0.45 M_{\odot}$ helium core at the tip of the red giant branch.
}

the dust extinction is largely produced in the ISM. A possible exception is the heavily-reddened SN 2006X, for which our measurement of the upper limit on the strength of the $5780 \AA$ DIB lies significantly below the $\mathrm{EW}(5780)$ versus $A_{V}$ relation in the Milky Way (see Figure 5). As mentioned in Section 4.3, SN 2006X showed variable Na I D absorption, and thus almost surely had CSM. Evidence in favor of the dust reddening being produced in the CSM of this SN was reported by Folatelli et al. (2010), who found that the reddening was better matched by a Goobar (2008) model than a normal reddening law.

Alternatively, the weak DIB absorption in SN 2006X may be a consequence of conditions in the ISM. A recent survey of sight-lines in the Scorpius OB2 association by Vos et al. (2011) showed that the correlation of the $5780 \AA$ A DIB with reddening is different for a diffuse environment exposed to a strong ultraviolet (UV) radiation field with low molecular content, as opposed to a denser cloud protected from the impinging interstellar UV radiation. The relations plotted in Figure 5 correspond more to the "UV exposed" trend, whereas the observations indicate that SN 2006X exploded behind a dense molecular cloud (Cox \& Patat 2008) which may have probed a "UV protected" environment characterized by a shallower dependence of $E W(5780)$ on $A_{V}$ (Vos et al. 2011). This may explain why SN 2006X falls below the general trend seen in Figure 5.

An independent test of the values of $R_{V}$ derived from the SN colors is provided by spectropolarimetric measurements, since the wavelength of maximum polarization is well-correlated with $R_{V}$ (Sarkowsky et al. 1975; Whittet \& van Breda 1978; Clayton \& Mathis 1988). Using this technique, Hough et al. (1987) found $R_{V}=2.4 \pm 0.23$ for SN $1986 \mathrm{G}$, which compares quite well with our value of $2.57_{-0.21}^{+0.23}$ derived from the optical and NIR light curves. Spectropolarimetry of SN 2001el by Wang et al. (2003) gave $R_{V}=2.88 \pm 0.15$, which is in fair agreement with our value of $2.25_{-0.36}^{+0.46}$. More recently, Patat et al. (2009) obtained spectropolarimetry of SN 2006X, for which the SN colors imply $R_{V}=1.31_{-0.10}^{+0.08}$. A strongly-polarized continuum was observed, peaking at $\lesssim 3500 \AA$, consistent with $R_{V} \lesssim 2$. Moreover, the angle of the interstellar polarization was found to be tangential to the dust lane associated with the spiral arm close to the explosion site, in keeping with observations that dust grains in the ISM of disk galaxies are aligned along the spiral arm pattern (e.g., Scarrott et al. 1987). These results imply that the majority of the dust reddening of SN 2006X was produced in the ISM.

We conclude, therefore, that the low values of $R_{V}$ observed for these reddened SNe Ia reflect the dust properties of the ISM in the neighborhood of the SN progenitor. The fact that we find $R_{V}<2$ for such a large fraction of reddened events is clearly telling us something important about both the environment and nature of the SN progenitors, many of which exploded in the dusty, inner regions of spiral galaxies. In turn, SN observations appear to provide a powerful new means of studying the extreme dust properties found in at least some spirals.

\section{CONCLUSIONS}

The observations in this paper lead us to two important conclusions about SNe Ia. The first of these is that the dust responsible for the observed reddening of SNe Ia appears to be predominantly located in the ISM of the host galaxies and not in CSM associated with the progenitor system. This conclusion is based on both the correlation found between the strength of the DIB at $5780 \AA$ and the visual extinction, $A_{V}$, derived from the 
SN colors, and the fact that DIBs are a characteristic of the ISM in our Galaxy, and not the CSM of mass losing stars. A direct implication of this finding is that the peculiarly-low values of $R_{V}$ derived for the most reddened SNe Ia are not generally due to multiple scattering of photons in a dusty CSM as suggested by Wang (2005) and Goobar (2008), but rather are characteristic of the properties of the dust in the ISM where the SNe exploded. Of course, we cannot rule out the presence of some dust in the CSM, but the evidence is consistent with the majority of reddening being produced in the host galaxy ISM.

The second major conclusion of this paper is that approximately one quarter of all SNe Ia show anomalously large host $\mathrm{Na}$ I column densities in comparison with the amount of dust that reddens their spectra. This result is based on comparison with the correlation between the $\mathrm{Na}$ I column density and $A_{V}$ observed in the Milky Way, but similar correlations are also observed for the Magellanic Clouds where the ISM is of significantly lower metallicity. Remarkably, all of the SNe with unusually strong D lines have "Blueshifted" profiles in the classification scheme of Sternberg et al. (2011). It is tempting, therefore, to ascribe the anomalous strength of the Na I to outflowing CSM, especially since strong D lines are observed in several SNe Ia that did not suffer significant dust reddening. We have identified ways of producing enhanced $\mathrm{Na}$ abundances in the CSM in both the SD and DD progenitor models. Nevertheless, unusually strong Na I $\mathrm{D}$ lines have not been observed in the few SNe Ia studied to date for which there is independent evidence for the existence of CSM, casting some doubt on the CSM interpretation.

Our observations show that the column density and/or equivalent width of the $\mathrm{Na}$ I $\mathrm{D}$ lines are, in general, unreliable indicators of the dust extinction suffered by SNe Ia. The exception to this statement is that weak or undetectable $\mathrm{Na}$ I absorption appears to be consistent with little or no extinction. We find that a better predictor of individual SN Ia extinction is the equivalent width of the DIB at $5780 \AA$, although this method requires moderate resolution $(\lambda / \Delta \lambda \gtrsim 4,000)$, high signal-to-noise ratio spectra, and yields host $A_{V}$ values with a precision of only $\pm 50 \%$.

Obviously there are many details of the CSM versus ISM puzzle left to resolve. Nevertheless, high-dispersion spectroscopy has revealed a number of critical clues to the nature of SN Ia progenitors, including the discovery of variable Na I absorption, the excess of blueshifted absorbers, the connection between blueshifted absorption and ejecta velocities, and now the correlation of blueshifted absorption with $\mathrm{Na}$-rich gas and the finding that the extinction affecting the $\mathrm{SN}$ light curves arises in the host galaxy ISM. Larger samples of such observations combined with optical and near-infrared light curves seem likely to shed further light on the physical mechanism responsible for these apparently related phenomena.

The work of the CSP has been supported by the National Science Foundation under grants AST0306969, AST0607438, and AST1008343. M.M.P. gratefully acknowledges the Aspen Center for Physics and NSF grant 1066293 for hospitality during the conception of this work. M.M.P. also thanks Brandon Lawton, Andy McWilliam, and Sebastián López for helpful discussions, and the Australian Astronomical Observatory and the ARC Centre of Excellence for All-sky Astrophysics (CAASTRO) for hosting and supporting a three month research leave during which this paper was completed. A.I.K. is grateful for support from the Australian Research Council for a Future Fellowship (FT110100475). A.G. was supported by the EU/FP7 via an ERC grant, a Minerva ARCHES prize, and the
Kimmel Award for innovative Investigation. M.S. acknowledges generous support provided by the Danish Agency for Science and Technology and Innovation realized through a Sapere Aude Level 2 grant. M.H. and G.P. are grateful for support from Millennium Center for Supernova Science (P10-064-F), with input from Fondo de Innovación para la Competitividad, del Ministerio de Economia, Fomento y Turismo de Chile. Computing resources used for this work were made possible by a grant from the Ahmanson Foundation. This research has made use of the NASA/IPAC Extragalactic Database (NED) which is operated by the Jet Propulsion Laboratory, California Institute of Technology, under contract with the National Aeronautics and Space Administration.

Facilities: Magellan:Clay (MIKE), KECK:I (HIRES), HET (HRS)

\section{REFERENCES}

Astier, P., Guy, J., Regnault, N., et al. 2006, A\&A, 447, 31

Bernstein, R., Shectman, S. A., Gunnels, S. M., Mochnacki, S., \& Athey, A. E. 2003, Proc. SPIE, 4841, 1694

Blondin, S., Prieto, J. L., Patat, F., et al. 2009, ApJ, 693, 207

Bloom, J. S., Kasen, D., Shen, K. J., et al. 2012, ApJL, 744, L17

Bouret, J.-C., Lanz, T., Hillier, D. J., et al. 2003, ApJ, 595, 1182

Burns, C., Stritzinger, M., Phillips, M. M., et al. 2011, AJ, 141, 19

Cardelli, J. A., Clayton, G. C., \& Mathis, J. S. 1989, ApJ, 345, 245

Clayton, G. C., \& Mathis, J. S. 1988, ApJ, 327, 911

Cohen, J. G., Briley, M. M., \& Stetson, P. B. 2005, AJ, 130, 1177

Cohen, J. G., \& Meléndez, J. 2005, AJ, 129, 303

Conley, A., Carlberg, R. G., Guy, J., et al. 2007, ApJ, 664, L13

Contreras, C., Hamuy, M., Phillips, M. M., et al. 2010, AJ, 139, 519

Cordiner, M. A., Cox, N. L. J., Evans, C. J., et al. 2011, ApJ, 726, 39

Cottrell, P. L., \& Da Costa, G. S. 1981, ApJL, 245, L79

Cox, N. L. J., \& Cordiner, M. A. 2008, in IAU Symp. 251, Organic Matter in Space, ed. S. Kwok (Cambridge: Cambridge Univ. Press), 237

Cox, N. L. J., Cordiner, M. A., Cami, J., et al. 2006, A\&A, 447, 991

Cox, N. L. J., Cordiner, M. A., Ehrenfreund, P., et al. 2007, A\&A, 470, 941

Cox, N. L. J., \& Patat, F. 2008, A\&A, 485, L9

Cox, N. L. J., \& Patat, F. 2013, A\&A, submitted

Cristallo, S., Straniero, O., Gallino, R., et al. 2009, ApJ, 696, 797

Dilday, B., Howell, D. A., Cenko, S. B., et al. 2012, Sci, 337, 942

D’Odorico, S., di Serego Alighieri, S., Pettini, M., et al. 1989, A\&A, 215, 21

Dufour, R. J. 1984, in IAU Symp. 108, Structure and Evolution of the Magellanic

Clouds, ed. S. van den Bergh \& K. S. de Boer (Dordrecht: Reidel), 353

Fitzpatrick, E. L., \& Massa, D. 2007, ApJ, 663, 320

Folatelli, G., Phillips, M. M., Burns, C. R., et al. 2010, AJ, 139, 120

Foley, R., Challis, P. J., Filippenko, A. V., et al. 2012a, ApJ, 744, 28

Foley, R., Simon, J. D., Burns, C. R., et al. 2012b, ApJ, 752, 101

Foley, R. J., \& Kasen, D. 2011, ApJ, 729, 55

Förster, F., González-Gaitán, S., Anderson, J., et al. 2012, ApJL, 754, L21

Friedman, S. D., York, D. G., McCall, B. J., et al. 2011, ApJ, 727, 33

Frogel, J. A., Gregory, B., Kawara, K., et al. 1987, ApJL, 315, L129

Galazutdinov, G. A., Lo Curto, G., \& Krełowski, J. 2008, MNRAS, 386, 2003

Galazutdinov, G. A., Musaev, F. A., Krełowski, J., \& Walker, G. A. 2000, PASP, 112,648

Ganeshalingam, M., Li, W., Filippenko, A. V., et al. 2010, ApJS, 190, 418

Gehrz, R. D., Truran, J. W., Williams, R. E., \& Starrfield, S. 1998, PASP, 110, 3 Goobar, A. 2008, ApJL, 686, L103

Gordon, K. D., Clayton, G. C., Misselt, K. A., et al. 2003, ApJ, 594, 279

Gratton, R. G., Bonifacio, P., Bragaglia, A., et al. 2001, A\&A, 369, 87

Gutiérrez, C., Folatelli, G., Pignata, G., et al. 2011, BAAA, 54, 109

Hamuy, M., Folatelli, G., Morrell, N. I., et al. 2006, PASP, 118, 2

Hamuy, M., Phillips, M. M., Maza, J., et al. 1995, AJ, 109, 1

Hamuy, M., Phillips, M. M., Suntzeff, N. B., et al. 1996, AJ, 112, 2391

Hamuy, M., Phillips, M. M., Suntzeff, N. B., et al. 2003, Natur, 424, 651

Heckman, T. M., \& Lehnert, M. D. 2000, ApJ, 537, 690

Herbig, G. H. 1993, ApJ, 407, 142

Hicken, M., Challis, P., Jha, S., et al. 2009, ApJ, 700, 331

Hicken, M., Challis, P., Kirshner, R. P., et al. 2012, ApJS, 200, 12

Ho, L. C., \& Filippenko, A. V. 1995, ApJ, 444, 165 (erratum 463, 818 [1996])

Hobbs, L. M. 1974, ApJ, 191, 381

Hobbs, L. M., York, D. G., Snow, T. P., et al. 2008, ApJ, 680, 1256

Hobbs, L. M., York, D. G., Thorburn, J. A., et al. 2009, ApJ, 705, 32 
Horesh, A., Kulkarni, S. R., Fox, D. B., et al. 2012, ApJ, 746, 21

Hough, J. H., Bailey, J. A., Rouse, M. F., \& Whittet, D. C. B. 1987, MNRAS, 227, P1

Howell, D. A. 2011, NatCo, 2, 350

James, G., François, P., Bonifacio, P., et al. 2004, A\&A, 414, 1071

Junkkarinen, V. T., Cohen, R. D., Beaver, E. A., et al. 2004, ApJ, 614, 658

Karakas, A. I. 2010, MNRAS, 403, 1413

Karakas, A. I., García-Hernández, D. A., \& Lugaro, M. 2012, ApJ, 751, 8

Karakas, A. I., \& Lattanzio, J. C. 2007, PASA, 24, 103

Kemp, S. N., Bates, B., Beckman, J. E., et al. 2002, MNRAS, 333, 561

Kessler, R., Becker, A. C., Cinabro, D., et al. 2009, ApJ, 185, 32

Krisciunas, K., Suntzeff, N. B., Candia, P., et al. 2003, AJ, 125, 166

Krisciunas, K., Suntzeff, N. B., Phillips, M. M., et al. 2004, AJ, 128, 3034 (erratum 130, 350 [2005])

Larson, K. A., \& Whittet, D. C. B. 2005, ApJ, 623, 897

Larson, K. A., Whittet, D. C. B., \& Hough, J. H. 1996, ApJ, 472, 755

Larson, K. A., Wolff, M. J., Roberge, W. G., et al. 2000, ApJ, 532, 1021

Le Bertre, T., \& Lequeux, J. 1993, A\&A, 274, 909

Luna, R., Cox, N. L. J., Satorre, M. A., et al. 2008, A\&A, 480, 133

Maeda, K., Benetti, S., Stritzinger, M., et al. 2010, Natur, 466, 82

Maeda, K., Leloudas, G., Taubenberger, S., et al. 2011, MNRAS, 413, 3075

Maguire, K., Sullivan, M., Patat, F., et al. 2013, MNRAS, 436, 222

Mandel, K. S., Narayan, G., \& Kirshner, K. P. 2011, ApJ, 731, 120

Mannucci, F., Della Valle, M., Panagia, N., et al. 2005, A\&A, 433, 807

Maoz, D., \& Mannucci, F. 2012, PASA, 29, 447

Matheson, T., Joyce, R. R., Allen, L. E., et al. 2012, ApJ, 754, 19

Mazzei, P., \& Barbaro, G. 2011, A\&A, 527, 34

Merrill, P. W., \& Wilson, O. C. 1938, ApJ, 87, 9

Moore, K., \& Bildsten, L. 2012, ApJ, 761, 182

Mowlavi, N. 1999, A\&A, 350, 73

Munari, U., \& Zwitter, T. 1997, A\&A, 318, 269

Nataf, D. M., Gould, A., Fouqué, P., et al. 2013, ApJ, 769, 88

Patat, F. 2005, MNRAS, 357, 1161

Patat, F. 2013, in IAU Symp. 281, Binary Paths to Type Ia Supernovae Explosions, ed. R. Di Stefano, M. Orio, \& M. Mo (Cambridge: Cambridge Univ. Press), 291

Patat, F., Baade, D., Höflich, P., et al. 2009, A\&A, 508, 229

Patat, F., Chandra, P., Chevalier, R., et al. 2007, Sci, 317, 924

Patat, F., Cordiner, M. A., Cox, N. L. J., et al. 2013, A\&A, 549, 62

Peimbert, M., \& Torres-Peimbert, S. 1976, ApJ, 203, 581

Pettini, M., \& Pagel, B. E. J. 2004, MNRAS, 348, L59

Phillips, M. M. 1993, ApJL, 413, L105

Phillips, M. M. 2012, PASA, 29, 434

Phillips, M. M., Phillips, A. C., Heathcote, S. R., et al. 1987, PASP, 99, 592

Poznanski, D., Ganeshalingam, M., Silverman, J. M., \& Filippenko, A. V. 2011, MNRAS, 415, L81

Poznanski, D., Prochaska, J. X., \& Bloom, J. S. 2012, MNRAS, 426, 1465

Ramírez, S. V., \& Cohen, J. G. 2003, AJ, 125, 224
Raskin, C., \& Kasen, D. 2013, ApJ, 772, 1

Richmond, M. W., \& Smith, H. A. 2012, JAVSO, 40, 872

Richmond, M. W., Treffers, R. R., Filippenko, A. V., et al. 1995, AJ, 109, 2121

Rolleston, W. R. J., Trundle, C., \& Dufton, P. L. 2002, A\&A, 396, 53

Sarkowsky, K., Mathewson, D. S., \& Ford, V. L. 1975, ApJ, 196, 261

Sarre, P. J. 2006, JMoSp, 238, 1

Scarrott, S. M., Ward-Thompson, D., \& Warren-Smith, R. F. 1987, MNRAS, 224, 299

Schlafly, E. F., \& Finkbeiner, D. P. 2011, ApJ, 737, 103

Schlegel, D. J., Finkbeiner, D. P., \& Davis, M. 1998, ApJ, 500, 525

Schweizer, F., Burns, C. R., Madore, B. F., et al. 2008, AJ, 136, 1482

Scolnic, D. M., Riess, A. G., Foley, R. J., et al. 2013, arXiv:1306.4050

Sembach, K. R., Danks, A. C., \& Savage, B. D. 1993, A\&AS, 100, 107

Shen, K. J., Guillochon, J., \& Foley, R. J. 2013, ApJ, 770, 35

Siluk, R. S., \& Silk, J. 1974, ApJ, 192, 51

Simon, J. D., Gal-Yam, A., Gnat, O., et al. 2009, ApJ, 702, 1157

Sneden, C., Ivans, I. I., \& Fulbright, J. P. 2004, in Carnegie Observatories Astrophysics Series, Vol. 4: Origin and Evolution of the Elements, ed. A. McWilliam \& M. Rauch (Cambridge: Cambridge Univ. Press), 170

Sollerman, J., Cox, N., Mattila, S., et al. 2005, A\&A, 429, 559

Sparks, W. B., Carollo, C. M., \& Macchetto, F. 1997, ApJ, 486, 253

Springob, C. M., Haynes, M. P., Giovanelli, R., \& Kent, B. R. 2005, ApJS, 160,149

Sternberg, A., Gal-Yam, A., Simon, J. D., et al. 2011, Sci, 333, 856

Stritzinger, M., Burns, C. R., Phillips, M. M., et al. 2010, AJ, 140, 2036

Stritzinger, M., Phillips, M. M., Boldt, L. N., et al. 2011, AJ, 142, 156

Tripp, R. 1998, A\&A, 331, 815

Tuairisg, S. Ó., Cami, J., Foing, B. H., Sonnentrucker, P., \& Ehrenfreund, P. 2000, A\&AS, 142, 225

Turatto, M., Benetti, S., \& Capellaro, E. 2003, in From Twilight to Highlight: The Physics of Supernovae, ed. W. Hillebrandt \& B. Leibundgut (Berlin: Springer), 200

Udalski, A. 2003, ApJ, 590, 284

Vallerga, J. V., Vedder, P. W., Craig, N., \& Welsh, B. Y. 1993, ApJ, 411, 729

Vos, D. A. I., Cox, N. L. J., Kaper, L., Spaans, M., \& Ehrenfreund, P. 2011, A\&A, 533, 129

Wang, L. 2005, ApJL, 635, L33

Wang, L., Baade, D., Höflich, P., et al. 2003, ApJ, 591, 1110

Wang, X., Wang, L., Filippenko, A. V., Zhang, T., \& Zhao, X. 2013, Sci, 340,170

Welsh, B. Y., Lallement, R., Vergely, J.-L., \& Raimond, S. 2010, A\&A, 510,54

Welty, D. E., Federman, S. R., Gredel, R., Thorburn, J. A., \& Lambert, D. L. 2006, ApJS, 165, 138

Welty, D. E., \& Hobbs, L. M. 2001, ApJS, 133, 345

Whittet, D. C. B., \& van Breda, I. G. 1978, A\&A, 66, 57

Yaron, O., \& Gal-Yam, A. 2012, PASP, 124, 668

York, B. A., Ellison, S. L., Lawton, B., et al. 2006, ApJL, 647, L29

Yuan, H. B., \& Liu, X. W. 2012, MNRAS, 425, 1763 